\title{
Traffic Flow Characteristics and Lane Use Strategies for Connected and Automated Vehicles in Mixed Traffic Conditions
}

\author{
Zijia Zhong $\mathbb{C D}^{1}{ }^{1}$ Joyoung Lee $\mathbb{C D}^{1}{ }^{1}$ and Liuhui Zhao $\mathbb{1}^{2}$ \\ ${ }^{1}$ John A. Reif, Jr. Department of Civil and Environmental Engineering, New Jersey Institute of Technology, NJ, USA \\ ${ }^{2}$ College of Engineering, Georgia Institute of Technology, GA 30332, Atlanta, USA \\ Correspondence should be addressed to Zijia Zhong; zijia.zhong@njit.edu
}

Received 22 April 2020; Revised 20 August 2020; Accepted 22 November 2020; Published 13 January 2021

Academic Editor: Meng Meng

Copyright (c) 2021 Zijia Zhong et al. This is an open access article distributed under the Creative Commons Attribution License, which permits unrestricted use, distribution, and reproduction in any medium, provided the original work is properly cited.

Managed lanes, such as a dedicated lane for connected and automated vehicles (CAVs), can provide not only technological accommodation but also desired market incentives for road users to adopt CAVs in the near future. In this paper, we investigate traffic flow characteristics with two configurations of the managed lane across different market penetration rates and quantify the benefits from the perspectives of lane-level headway distribution, fuel consumption, communication density, and overall network performance. The results highlight the benefits of implementing managed lane strategies for CAVs: (1) A dedicated CAV lane significantly extends the stable region of the speed-flow diagram and yields a greater road capacity. As the result shows, the highest flow rate is 3400 vehicles per hour per lane at $90 \%$ market penetration rate with one CAV lane. (2) The concentration of CAVs in one lane results in a narrower headway distribution (with smaller standard deviation) even with partial market penetration. (3) A dedicated CAV lane is also able to eliminate duel-bell-shape distribution that is caused by the heterogeneous traffic flow. (4) A dedicated CAV lane creates a more consistent CAV density, which facilitates communication activity and decreases the probability of packet dropping.

\section{Introduction}

The mobility landscape is experiencing a paradigm shift due to rapid advancements of the information and vehicular technologies. Among them, the connected and automated vehicle (CAV) technologies have been contributing to the adoption of next-generation vehicles that are equipped with connectivity (i.e., connected vehicles) and/or automation (i.e., automated vehicles). In spite of CAV's immense benefits and potentials in reshaping the mobility landscape, the adoption of CAVs by consumers is still uncertain [1], although some lower-level vehicle automation in the form of driver-assistance system has been commercially available.

The near-term deployment of CAVs is characterized by mixed traffic conditions, where human-driven vehicles (HVs) and CAVs constantly interact with each other. The potential benefits from CAVs may be offset by the interactions among different types of vehicles. For example, the short following time gap (e.g., $0.6 \mathrm{~s}$ ) is only feasible when a CAV follows another CAV. To overcome such shortcoming in near-term CAV deployment, managed lane strategies, such as CAV dedicated lane, are one of the promising solutions in order to facilitate the formation of the CAV strings. Practically, managed lane strategies are freeway lanes that are set aside and operated under various fixed and/or real-time strategies in response to certain objectives, such as improving traffic operation [2]. It is also anticipated that managed lane strategies incentivize the adoption of CAV, just as they did for encouraging car-pooling or low emission vehicles.

The goal of this study is to investigate the impact of different lane use strategies under mixed traffic conditions at vehicle trajectory, as well as lane, level. For clarity, we refer mixed traffic condition to the condition that CAVs and HVs operate on the same roadway network in the following discussions. The contributions of the paper include the following: 
(1) The analysis of CAV-enhanced traffic flow characteristics at the lane level and vehicle level

(2) The investigation of traffic performance with gradual introduction of CAV platoons under difference managed lane strategies

(3) The implications of managed lane strategies from a dedicated short-range communication (DSRC) perspective

The remainder of the paper is organized as follows. Related work regarding the research of CAVs in mixed traffic and managed lanes is reviewed in Section 2, followed by the evaluation methodology, including customized CAV module and defined scenarios, in Section 3. The simulation results are presented and discussed in Section 4. Lastly, findings and recommendations are discussed in Section 5.

\section{Literature Review}

There have been numerous studies on the implementation and evaluation of CAVs in various traffic settings. Aligning with our research topic, we focused our literature search on two key aspects of CAV studies: (1) CAV evaluation in mixed traffic conditions at network level and (2) managed lane strategies for CAV. A list of abbrevations used can be found in Table 1 in the Appendix.

2.1. CAVEvaluation in Mixed Traffic Conditions. Three main approaches have been used to assess the benefits of CAVs: (1) analytical study, (2) simulation evaluation, and (3) field test with equipped vehicles.

On-road testing provides the utmost degree of realism with equipped automated driving systems (ADS) and realworld traffic environment. However, the safety and efficiency issues for testing CAV on public roads have been the major concern, especially after several severe CAV-involved accidents in recent years. Due to safety, technological, and budgetary limitations, the scale of a CAV field test at current stage tends to be small (e.g., with a handful of CAVs). As a result, the conclusions from these small-scale field tests may not be reliably generalized to a traffic flow level. Furthermore, it was estimated by Kalra and Paddock that billions of kilometers of road test would be required to achieve the desired level of confidence in terms of safety of an ADS [3]. Thus, analytical and simulation approaches serve as two primary methods for evaluating traffic flow impact of CAVs.

The majority of the analytical models is based on macroscopic traffic flow models and may experience difficulty in faithfully capturing the complex phenomena in transportation networks, such as lane drop. Smith et al. proposed an analytical framework for assessing the benefits of CAV operations [4]. The results indicated that CAVs improved network mobility performance, even with low MRP and no managed lane policies. Throughput, without managed lanes increased by $4 \%, 10 \%$, and $16 \%$ at the MPR of $10 \%, 20 \%$, and $30 \%$, respectively. It was also discovered that the managed lane policy
TABLE 1: List of abbreviations.

\begin{tabular}{lc}
\hline Abbreviation & Definition \\
\hline ADAS & Advanced driver-assistance systems \\
ADS & Automated driving systems \\
ACC & Adaptive cruise control \\
AV & Automated vehicles \\
API & Application programming interface \\
BSM & Basic safety message \\
CV & Connected vehicles \\
CAV & Connected and automated vehicles \\
CACC & Cooperative adaptive cruise control \\
CAH & Constant-acceleration heuristic \\
CDF & Cumulative probability function \\
CHEM & Comprehensive modal emission model \\
DSRC & Dedicated short-range communication \\
DLL & Dynamic-link library \\
DTG & Desired time gap \\
E-IDM & Enhanced intelligent driver model \\
GPL & General purpose lane \\
HV & Human-driven vehicle \\
HOV & High-occupancy vehicles \\
IEEE & Institute of Electrical and Electronics Engineers \\
MPR & Market penetration rate \\
MOVES & Motor vehicle emission simulator \\
PET & Postencroachment time \\
SSAM & Surrogate safety assessment model \\
SAE & Simulation of urban mobility \\
SUMO & Time to collision \\
TTC & Vehicle awareness device \\
VAD & Wireless access in vehicular environment \\
WAVE & \\
\hline &
\end{tabular}

facilitated homogeneous CAV traffic flow, leading to more consistent and stable network outputs. An analytical model for determining the optimal managed lane strategy was proposed in [5], where the maximum system throughput in a mixed traffic condition could be calculated under the assumption of random vehicle distribution on a freeway facility. Three types of headways (i.e., conservative, neutral, and aggressive) were used in the model. Wang et al. proposed a second-order traffic flow model for mixed traffic streams with HVs and AVs. The authors found that the second-order model consistently outperformed the first-order one in terms of the accuracy of traffic density when the variability of the penetration rate increased [6].

At the corridor level, a capacity of $4250 \mathrm{vph} / \mathrm{pl}$ (vehicle per hour per lane) was observed in [7] on a $6 \mathrm{~km}$ highway segment with uniformly distributed ramps under full market penetration of CAVs. The study by Shladover et al. observed a pipeline capacity of 3600 at $90 \%$ MPR of CAVs [8], where the pipeline capacity refers to the throughput observed on a single-lane roadway without any interference of lateral movements [9].

Arnaout and Arnaout evaluated CAVs under moderate, saturated, and oversaturated demand levels on a hypothetical 4-lane highway under different market penetrations. They found that 9400 vehicles could be served within an hour when the CAV MPR reached $40 \%$ [10]. Songchitruksa et al. assessed the network 
performance with CAVs on the 26-mile I-30 freeway in Dallas, TX, and found the highest throughput being $4400 \mathrm{vph}$ at $50 \%$ MPR [11] among four MPR scenarios (i.e., $10 \%, 30 \%, 50 \%$, and $70 \%$ ). Another study [12] also revealed that the mobility benefits of CAV emerged at $30 \%$ MPR.

Liu et al. investigated the benefits of alleviating freeway merge bottleneck and compared the performance of CACC with ACC under full market penetration. The results showed that CACC yielded a 50\% reduction in fuel consumption (as estimated with the EPA MOVES model) while increasing corridor capacity by $49 \%$, compared to the ACC scenario [13]. With a subsequent test on an $18 \mathrm{~km}$ segment of SR-99, the research team found that deploying vehicle awareness device- (VAD-) equipped vehicles along with managed lane strategies was helpful in improving corridor-level traffic flow under low and medium CAV market penetrations [14]. Besides MOVES, comprehensive modal emission model (CMEM) [15], VT-Micro [16], and the Future Automotive Systems Technology Simulator (FASTSim) [17] are among the commonly used vehicle emission models in quantifying potential environmental impact of deploying CAVs.

The potential impact of the short following time headway of CAVs on HVs has also been studied in previous studies. Among them, the KONVOI project found the carry-over effect for CACC drivers in manual driving after the disengagement of the CACC system [18]. In recent years, driving simulator has been employed to study the behavioral adaptation of human drivers operating in the vicinity of CAVs. Nowakowski et al. found that test participants are likely to drive under a shorter following distance in the presence of CACC platoons in the adjacent lane [19]. A similar study was conducted by Gouy et al. to investigate the behavioral adaptation of human drivers along a CACC platoon, in which two CACC platoon configurations were tested: (1) a 10-truck platoon with $0.3 \mathrm{~s}$ intraplatoon headway and (2) a 3-truck platoon with $1.4 \mathrm{~s}$ intraplatoon headway. It was found that a smaller average HV headway was observed in the first scenario, under which participants spent more time under a $1 \mathrm{~s} \mathrm{headway,} \mathrm{although} \mathrm{such} \mathrm{short}$ headway was generally deemed unsafe in previous studies (e.g., [20]).

2.2. CAVs and Managed Lanes. Managed lanes have been in practice over the years to improve target operation objectives, such as (1) promoting the adaptation of environment-friendly vehicles by offering priority usage to specific travel lanes (e.g., the California Clean Air Vehicle Decal [21]), (2) encouraging car-pooling by adopting highoccupancy vehicle (HOV) lanes [22], and (3) performing active traffic management with the aid of high-occupancy toll (HOT) lanes [23]. A CAV lane is one variant of managed lane strategies which provides exclusive lane use privilege to CAVs. Although managed lane strategies have been widely applied to highway operation with successful cases, due to distinctive operational characteristics of
CAVs, knowledge learned from a conventional managed lane may not be directly transferable to the implementation of a CAV lane.

The provision of a CAV-managed lane has two primary reasons. First, CAV-managed lanes can incentivize the adaptation of CAVs by offering priority usage to managed lanes, which typically provides better and more reliable travel because of active traffic management. More importantly and unique to CAVs, CAV-managed lanes can provide accommodations for the underlying operational characteristics of CAVs. A CAV is able to operate at a much closer headway than a human driver with the assistance of $\mathrm{V} 2 \mathrm{~V}$ wireless communication and the automated driving system (ADS) $[24,25]$. Hence, the necessary condition for realizing such a short following headway is the availability of the vehicle driving information of at least one of the predecessors on the same lane, that is, through a CAV following another CAV. Otherwise, the string stability of CAVs cannot be guaranteed [26], and the lack of thereof is termed as CAV degradation [27], which could potentially be a major hurdle for CAVs operating in mixed traffic. A numerical example by Wang et al. has showed that the current technological maturity of CACC contributed negatively to the stability of heterogeneous flow [27].

To mitigate CAV degradation, ad hoc coordination, local coordination, and global coordination are the three major strategies that outline the organization of CAV platoons [28]. Ad hoc coordination assumes that CAVs arrive in random sequence and do not actively seek clustering opportunities in a traffic stream. By extension, the probability of driving around other CAVs is highly correlated to MPR. On the contrary, CAVs actively identify and approach an existing CAV cluster (or other free-agent CAVs) to form a new cluster through local coordination, regardless of CAV MPRs. Finally, global coordination (a.k.a. end-to-end platooning) requires a high-level route planning and extensive communication to coordinate vehicles traveling with the same origin-destination pair even before the CAVs enter highway sections [29].

To successfully form and maintain platoons, accurate and cost-effective localization of CAVs in a dynamic traffic environment remains one of the biggest challenges, especially for local coordination $[28,30]$. In the presence of a CAV-managed lane, a higher concentration of CAVs facilitates local coordination with much less stringent requirements on the accuracy of vehicle localization. In addition, the CAV-managed lane strategy aligns well with the three-stage deployment roadmap considering market diffusion and technological maturation for CAVs [31]. In the first stage, the adoption of CAVs is incentivized by allowing the use of the managed lane free of charge. At this stage, the following headway of CAVs on the managed lane may be comparable to that which has been observed from HVs for safety reasons in a mixed traffic condition. In the second stage, a shorter following headway for CAVs could be implemented to further increase the carrying capacity of the managed lane when the demand of CAVs along with the familiarity of road users to CAVs increases. 
In the third stage, when the CAVs reach a critical level of MPR, high-performance driving enabled by the CAVs can be achieved due to homogeneous CAV traffic flow on the managed lane.

To assess the impact of CAV-managed lane strategies, Zhang et al. compared the performance of a managed lane and general propose lanes (GPL) based on average speed, throughput, and travel time [32]. The results indicated that the speed improvement in the managed lane was significant compared to that of GPLs. With $20 \%$ MPR, the latent demand (the demand that cannot enter the simulation network due to congestion) decreased to zero. Inspired by the fluid approximation of traffic, Wright et al. proposed an algorithm for simulating the weaving activity at the interface of a managed lane and the adjacent GPL at a macroscopic scale [33]. Chen et al. proposed a time-dependent deployment framework that was formulated with a network equilibrium model and a diffusion model. With the constraint of a given set of candidate lanes which corresponds to the field condition, the social cost was minimized with the consideration of different MPR levels [34]. Zhong and Lee studied four managed lane strategies and compared the benefits for GPL and managed lane users in terms of mobility, safety, emission, and equity [35]. In freeway settings, the authors recommended a $30 \%$ minimal MRP for deploying a CAVmanaged lane to avoid lane use imbalance that could degrade the performance $[31,36]$.

Qom et al. proposed a multiresolution framework to study the mobility impact of CAV lanes. Traffic flow-based static traffic assignment and the mesoscopic simulationbased dynamic traffic assignment were adapted in the bilevel framework. The former yielded the MPR-based trends, whereas the latter refined the trend based on traffic congestion. The results indicated that it was not beneficial to provide toll incentive for CAVs at lower MPR due to the marginal increase in highway capacity [37]. Ghiasi et al. proposed an analytical capacity model for mixed traffic [38]. The model relied on the Markov chain representation of the spatial distribution of heterogeneous and stochastic headway. With the sufficient and necessary condition of capacity increase proven, the authors emphasized the importance of quantitative analysis of the actual headway setting.

The introduction of a CAV lane to a signalized corridor was reported in [39]. Two configurations of a CAV lane, along with other managed lanes, were evaluated. To accommendate for the turning movements, buffer zones were implemented, where HVs are allowed to temporarily use the CAV lane. Papadoulis et al. evaluated the safety impact of CAVs using the Surrogate Safety Assessment Model (SSAM) [40]. The time to collision (TTC) and the postencroachment time (PET) were adapted with safety thresholds of $1.5 \mathrm{~s}$ and $5 \mathrm{~s}$, respectively. They observed substantial safety benefits in terms of reduction in traffic conflicts: $12-47 \%$ at $25 \%$ MPR to $90-94 \%$ at $100 \%$ MPR. In [41], TTC was also used to assess the safety conditions for
HVs when CAV local clustering strategy was employed. Ali et al. found that drivers with advanced traffic information enabled by connectivity tend to wait longer and maintained a larger space on mandatory lane change (the communication delay for lane merging assistance was unnoticeable when it was less than $1.5 \mathrm{~s}$ ). Postencroachment time (PET) analysis also indicated improved travel safety from CAV implementation [42].

2.3. Summary. The vast majority of previous studies evaluated the benefits of CAVs at an aggregated level with the emphasis of overall traffic improvement. Analytic models are in macroscopic nature under overly ideal conditions, and they have difficulty in factoring the stochastic nature of human drivers in a mixed traffic environment. CAVmanaged lane strategy could be instrumental in the nearterm deployment of CAVs, but it is still an underexplored area, despite its increasing recognition.

\section{Evaluation Framework and Experiment Design}

This study focuses on analyzing mixed traffic flow characteristics at a corridor level considering different CAV MRPs and managed lane strategies. In this section, the integrated simulation test bed, transportation network, and simulation scenarios are discussed in detail.

3.1. CAV Behavior Model. The PTV Vissim [43], a commercial-off-the-shelf microscopic simulation package, is chosen for the evaluation. Vissim has been widely adapted by transportation practitioners and researchers, owing to its high-fidelity simulation mechanism and flexible modules. Compared to other open-source traffic simulators (e.g., SUMO), one reservation for Vissim being a commercial software is its close-sourced nature. As shown in Table 2, a calibrated Wiedemann car-following model and the enhanced intelligent driver model (E-IDM) [44] were used to model HVs and CAVs, respectively. The intelligent driver model (IDM) and its variants have been used to design the ACC/CACC controller that resembles human-like car-following behaviors [45-49]. As an improved iteration of the collision-free IDM [50], the E-IDM deals with CAV longitudinal maneuver. The behavior model of the E-IDM is expressed in equations (1)-(3): where $a$ is the maximum acceleration; $b$ is the desired deceleration; $c$ is the coolness factor; $\delta$ is the free acceleration exponent; $\dot{x}$ is the current speed of the subject vehicle; $\dot{x}_{\text {des }}$ is the desired speed, $\dot{x}_{\text {lead }}$ is the speed of the lead vehicle; $s_{0}$ is the minimal distance; $\ddot{x}$ is the acceleration of the subject vehicle; $\ddot{x}_{\text {lead }}$ is the acceleration of the lead vehicle; $\ddot{x}_{\text {IDM }}$ is the acceleration calculated by the original IDM model [50]. The minimal distance can be calculated as $s^{*}\left(\dot{x}, \dot{x}_{\text {lead }}\right)$, 
TABLE 2: Differences between HVs and CAVs in the simulation models.

\begin{tabular}{lccc}
\hline Vehicle type & Longitudinal control & DTG & Stochasticity \\
\hline HV & Wiedemann 99 & $1.4 \mathrm{~s}$ & Y \\
CAV & E-IDM & $0.6,1.2 \mathrm{~s}$ & $\mathrm{~N}$ \\
\hline
\end{tabular}

$$
\begin{aligned}
& \ddot{x}= \begin{cases}a\left[1-\left(\frac{\dot{x}}{x_{\mathrm{des}}}\right)^{\delta}-\left(\frac{s^{*}\left(\dot{x}, \dot{x}_{\text {lead }}\right)}{s_{0}}\right)\right], & \text { if } \ddot{x}_{\mathrm{IDM}} \geq \ddot{x}_{\mathrm{CAH}}, \\
(1-c) \ddot{x}_{\mathrm{IDM}}+c\left[\ddot{x}_{\mathrm{CAH}}+b \cdot \tanh \left(\frac{\ddot{x}_{\mathrm{IDM}}-\ddot{x}_{\mathrm{CAH}}}{b}\right)\right], & \text { otherwise, }\end{cases} \\
& s^{*}\left(\dot{x}, t \dot{x}_{\text {lead }}\right)=s_{0}+\dot{x} T+\frac{\dot{x}\left(\dot{x}-t \dot{x}_{\text {lead }}\right)}{2 \sqrt{a b}}, \\
& \ddot{x}_{\mathrm{CAH}}= \begin{cases}\frac{\dot{x}^{2} \cdot \min \left\{\ddot{x}_{\text {lead }}, \ddot{x}\right\}}{\dot{x}_{\text {lead }}^{2}-2 x \cdot \min \left\{\ddot{x}_{\text {lead }}, \ddot{x}\right\}}, & \text { if } \dot{x}_{\text {lead }}\left(\dot{x}-\dot{x}_{\text {lead }}\right) \leq-2 x \cdot \min \left\{\ddot{x}_{\text {lead }}, \ddot{x}\right\}, \\
\min \left\{\ddot{x}_{\text {lead }}, \ddot{x}\right\}-\frac{\left(\dot{x}-\dot{x}_{\text {lead }}\right)^{2} \Theta\left(\dot{x}-\dot{x}_{\text {lead }}\right)}{2 x}, & \text { otherwise. }\end{cases}
\end{aligned}
$$

where $T$ is the desired time gap; and $\ddot{x}_{\mathrm{CAH}}$ is the acceleration calculated by the constant-acceleration heuristic (CAH) component, where $\Theta$ is the Heaviside step function that is used to eliminate the negative approaching rate of subject vehicle [44].

In this study, the E-IDM model is selected as the longitudinal control for the CAVs. Although without built-in multianticipative car-following function, as the literature shows, E-IDM is still a good simple car-following model for CAVs, as the stochastic nature of human driving is removed (i.e., automation property), and the acceleration of the preceding vehicle is taken into account in the driving model (i.e., connectivity property). As shown in Table 3, all the parameters remain the same as those originally specified in [44], with the exception of the desired time gap (DTG), which is defined with two values: $0.6 \mathrm{~s}$ and $1.2 \mathrm{~s}$. The former DTG is used when the communication between a preceding CAV and the subject CAV is successful, whereas the latter one is in effect when the communication failure occurs. The updating frequency for the E-IDM model in Vissim is $10 \mathrm{~Hz}$. The density of CAVs which is used to calculate the communication activity is updated at a $2 \mathrm{~Hz}$ frequency to reflect the traffic dynamic. Each transmission is assumed to have up to five attempts (four retransmissions). At least one successful attempt is required for a transmission to be considered successful, upon which the DTG is determined.

To implement these two car-following models in Vissim, the subset of the human driving behavior is realized by adjusting car-following parameters of the Wiedemann carfollowing model, which is relatively straightforward. The E-IDM, on the other hand, is implemented via the external driver model application programming interface (API) and connected with Vissim through a dynamic link library (DLL). The DLL is invoked in each simulation time step such that the default car-following behavior will be overwritten for a specified vehicle type. The DSRC wireless communication module, discussed later in Section 3.2, is also implemented in the API to achieve a dynamic response based on prevailing traffic conditions.

One of the most prominent features in CAV behavior modeling is the short time headway during car-following, which is manifested by several key differences between a $\mathrm{CAV}$ and a HV. First, the stochasticity of the CAVs is significantly lower than that of human drivers. This is enabled by the on-board sensors that are able to continuously and accurately perceive the surrounding environment. However, the stochasticity cannot be completely eliminated due to sensor noise and communication delay/error. Second, a CAV has minimal reaction time due to its algorithmic decision-making process and computational power. Past studies have already identified the impact of the reaction time of human drivers in various traffic phenomena, including capacity drop [51] and flow stability [47], whereas driving simulation tests revealed that the information augmented by connectivity could decrease the reaction time for drivers [52].

In addition, human factor plays a crucial role in the resumption of control of a CAV when an ADS exits its operational domain (e.g., high risk of collision, sensor failure, and communication interference). Quantitative evidence regarding the transition of control from traffic psychology or human-machine interactions is still limited [53], though few frameworks have been proposed to simulate human behavior endogenously $[54,55]$. For example, the prospect theory was used to model the risk and human perception [56, 57]. The Risk Allostasis Theory [58] was adopted for modeling relationship between cognitive processing of information and physical performance. The Task Capacity Interface [59] was employed by Saifuzzaman et al. for quantifying situational awareness of a driver. 
TABLE 3: E-IDM vehicle control parameters.

\begin{tabular}{lcccccccc}
\hline Parameter & $T_{\text {intra }}$ & $T_{\text {inter }}$ & $s_{0}$ & $a$ & $b$ & $c$ & $\theta$ & $\dot{x}_{\text {des }}$ \\
\hline Value & $0.6 \mathrm{~s}$ & $1.2 \mathrm{~s}$ & $1 \mathrm{~m}$ & $2 \mathrm{~m} / \mathrm{s}^{2}$ & $2 \mathrm{~m} / \mathrm{s}^{2}$ & 0.99 & 4 & $105 \mathrm{~km} / \mathrm{h}$
\end{tabular}

Calvert and van Arem developed a framework that encompasses the driving task demand and driver task saturation [53]. The framework's main goal is to assess the performance impact during the transition of control for AVs. The total task demand, situational awareness, and reaction time during the transition of control from AVs were explored. The framework showed promising capability in capturing the interactive effects of humans with lower-level AVs. However, empirical evidence is still needed to relax the assumptions used in the framework not only from the cognitive point of view but also from vehicle dynamics and intervehicle interactions.

Another human factor is driver compliance to the ADS. Since, in lower or medium level of automation, the driver is ultimately responsible for his or her vehicle, which means overwriting, when deemed necessary, is possible by the human driver, this control authority, in extreme cases, could cancel out the benefits promised by the CAV technologies. In a recent study [57], Sharma et al. employed the prospect theory to model driver decision-making mechanisms including irrational ones and captured the negative relationship between headway and compliance decision by a driver.

In this study, we represent the differences of a CAV and a $\mathrm{HV}$ with different desired time headways through separate car following models, with the following assumptions made for CAVs: (1) no error for the on-board sensors and the vehicle controller, that is, perfect perception; (2) no human factor modeling pertaining to the transition of authority; and (3) no behavior adaptation for CAVs for non-CAV drivers.

3.2. Wireless Communication Model. In an early study, we implemented a packet-level communication module through Vissim API [36]. Similar adaptations for the model were also found in previous studies $[11,36,60]$. The analytical model [61] was developed from ns-2, an empirical packet-level network simulator that returns the probability of one-hop broadcast reception of basic safety message (BSM) under IEEE 802.11p, an approved amendment tailored to wireless access in vehicular environment (WAVE) in the 802.11 family protocol. The model uses the concept of communication density level, a metric representing channel load in vehicular communication in the form of the sensible transmission per unit of time and per unit of the road [62]. The data reception rate is determined jointly by communication density level and transmission power. An illustration for the reception probability is shown in Appendix B. Note that this communication model only pertains to the physical layer of the DSRC communication (e.g., no MAC layer delay):

$$
\begin{aligned}
P_{r}(x, \delta, \varphi, f) & =e^{-3(x / \varphi)^{2}}\left(1+\sum_{i=1}^{4} h_{i}(\xi, \varphi)\left(\frac{x}{\varphi}\right)^{i}\right), \\
\xi & =\delta \cdot \varphi \cdot f,
\end{aligned}
$$

where $h_{i}(\xi, \varphi)$ is the two-dimensional polynomial of fourthdegree for all curving fitting parameters [63], which is also shown in Appendix B; $\xi$ is communication density, events/s/ $\mathrm{km}$; and $\varphi$ is the transmission power, $\mathrm{m} ; \delta$ is vehicle per kilometer that periodically broadcast messages, veh $/ \mathrm{km}$; and $f$ is transmission rate, $\mathrm{Hz}$.

3.3. Transportation Network. A $9.3 \mathrm{~km}$ 4-lane hypothetical network was constructed in Vissim with two interchanges located at mile markers $2(\mathrm{~km})$ and $6(\mathrm{~km})$, respectively. An abstract geometry of the network along with vehicle demand of the origins and destination is shown in Figure 1. The primary reason for using a simply synthetic network is to limit variables for the simulation. Note that the driving behavior parameters for the Wiedemann carfollowing model (for HVs) are the same as those in previous studies [31, 41,64,65], representing a subset of the calibrated driving behavior in the I-66 segment in northern Virginia. The demand originated on the mainline is deliberately set higher than usual to create a congested network. The speed limit for the mainline of the network is set as $120 \mathrm{~km} / \mathrm{h}$. Three data collectors are placed at "C1," "C2," and "C3" locations.

3.4. Managed Lane Scenarios. Three cases of CAV lanes, as shown in Table 4, are implemented in the network:

(1) No managed lane (NML): This scenario serves as the base condition of the study. There is no priority lane use for CAVs, and they are mixed with HVs throughout the network;

(2) One CAV lane (CAV-1): In this strategy, one CAV lane is implemented in the left-most lane (the fourth lane from the right);

(3) Two CAV lanes (CAV-2): An additional CAV lane is added to the CAV-1 case, making two CAV lanes available at the left-most lane and the second-left-most lane in the roadway segment. It aims to investigate the duel managed lane configuration.

As revealed in previous studies [31, 32, 66, 67], a managed lane may have a detrimental effect on traffic performance if implemented prematurely, that is, usually with an MPR less than 30\%. Therefore, in this study, we set CAV MPRs for "CAV-1" to start from 30\%. With the same logic, the "CAV-2" cases start with $40 \%$ to cover certain transition MPR, since the linear extrapolation may not hold. 


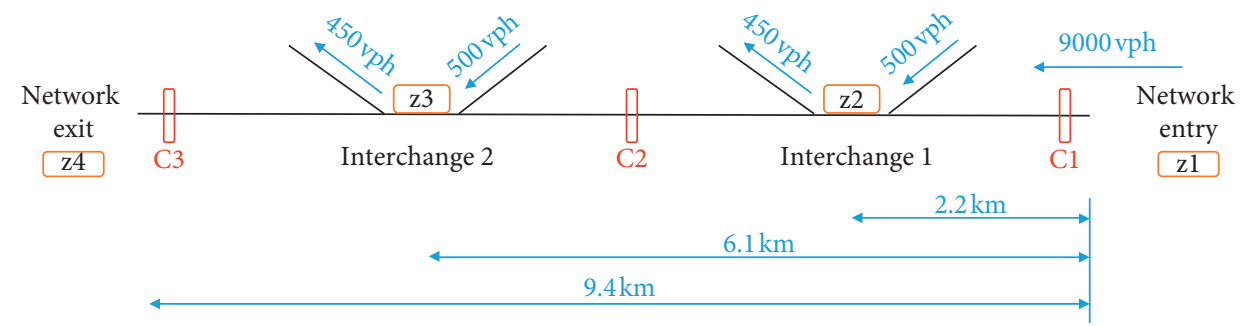

FIGURE 1: Network geometry and demand.

TABle 4: Managed lane evaluation plan.

\begin{tabular}{lccc}
\hline $\begin{array}{l}\text { Policy } \\
\text { ID }\end{array}$ & $\begin{array}{c}\text { No managed lane } \\
\text { NML }\end{array}$ & $\begin{array}{c}\text { Managed lane \#1 } \\
\text { CAV-1 }\end{array}$ & $\begin{array}{c}\text { Managed lane \#2 } \\
\text { CAV-2 }\end{array}$ \\
\hline 1st lane & HV + CAV & HV + CAV & HV + CAV \\
2nd lane & HV + CAV & HV + CAV & HV + CAV \\
3rd lane & HV + CAV & HV + CAV & CAV \\
4th lane & HV + CAV & CAV & CAV \\
MPR & $0 \%-100 \%$ & $30 \%-100 \%$ & $40 \%-100 \%$ \\
\hline
\end{tabular}
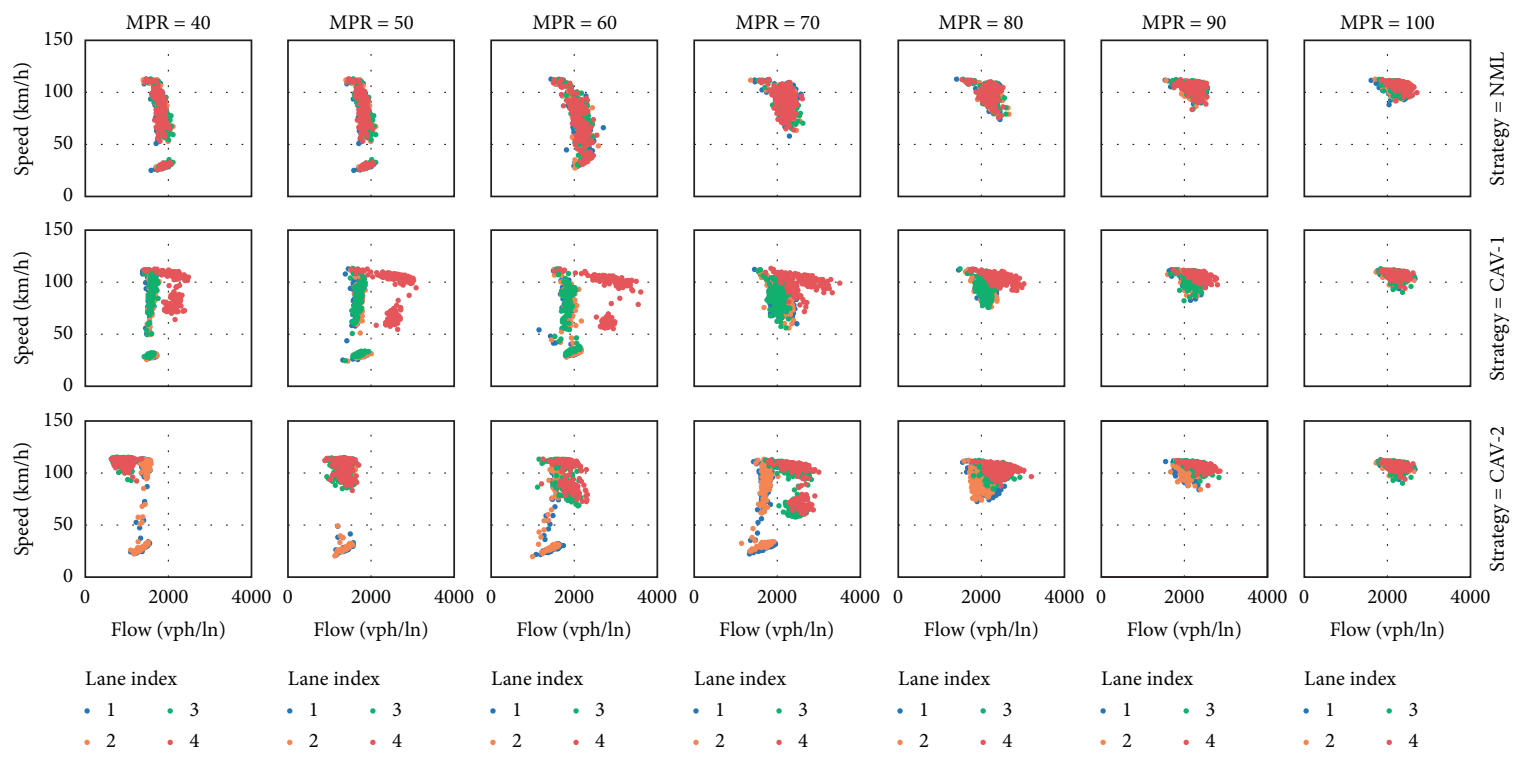

Lane index

Lane index

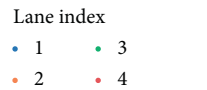

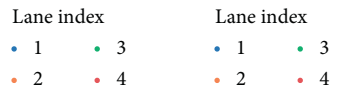

FIgURe 2: Speed-flow curves.

\section{Results and Analysis}

Five replications are run for each combination of managed lane policies and MPRs. Aggregated data are collected at 5minute intervals, and the raw data are collected at each simulation time step. The analysis is performed on five aspects: (1) traffic flow characteristics, (2) headway distribution, (3) fuel consumption, (4) wireless communication, and (5) overall network performance.

4.1. Traffic Flow Characteristic. Figure 2 exhibits the speedflow characteristics of the simulation scenarios having $40 \%$ MPR and above. The plot is color-coded by travel lanes with index " 1 " representing the right-most lane and " 4 " the leftmost lane. The speed-flow diagram is comprised of a stable region and an unstable (congested) region, separated by the optimum (maximum) flow. Several distinctive patterns can be observed. First, regardless of the managed lane strategy, the sample points become more concentrated as the MPR increases, with the disappearance of the congested region typically found in the lower speed region. Second, the CAV lane has a distinct pattern compared to the GPLs. Such pattern is most apparent in CAV-1, where the traffic samples on the left-most lane (CAV lane) shift to the right along the flow axis. The congested region disappears when MPR reaches $70 \%$ in the CAV-1 case for all of the lanes. The improvement for the GPs is due to a higher carrying capacity of the CAV lane, which results in less traffic on the GPLs. The homogeneity of the CAV traffic is the primary factor in realizing the mobility benefit of CAVs: in NML cases, the sample points from different lanes are evenly distributed, in 

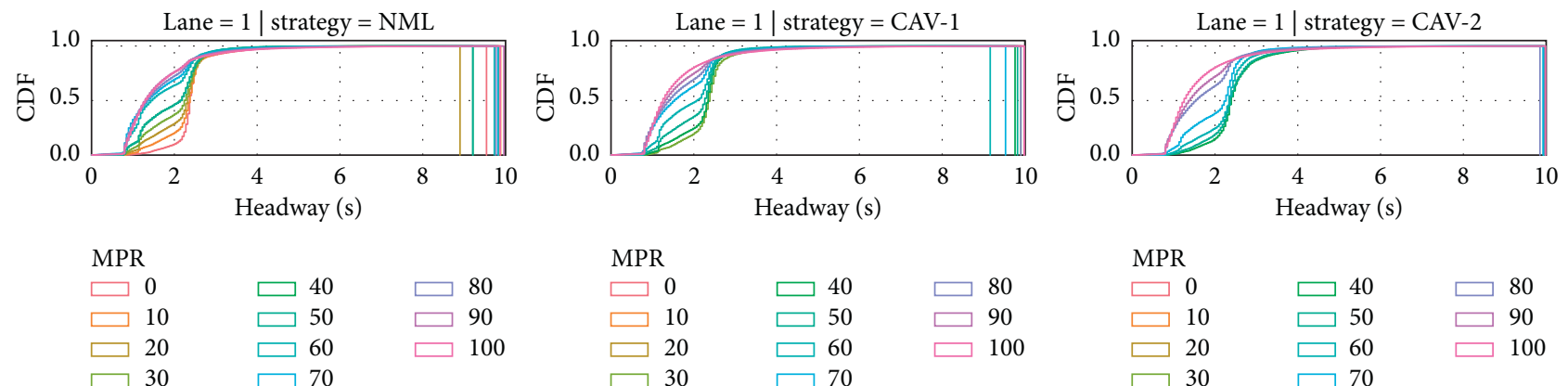

MPR

$\square 0$

$\square 10$

$\square 40$

$\square 80$

$\square 20$

$\square 50$

$\square 90$

$\square \quad 20$

$\square 60$

$\square 100$
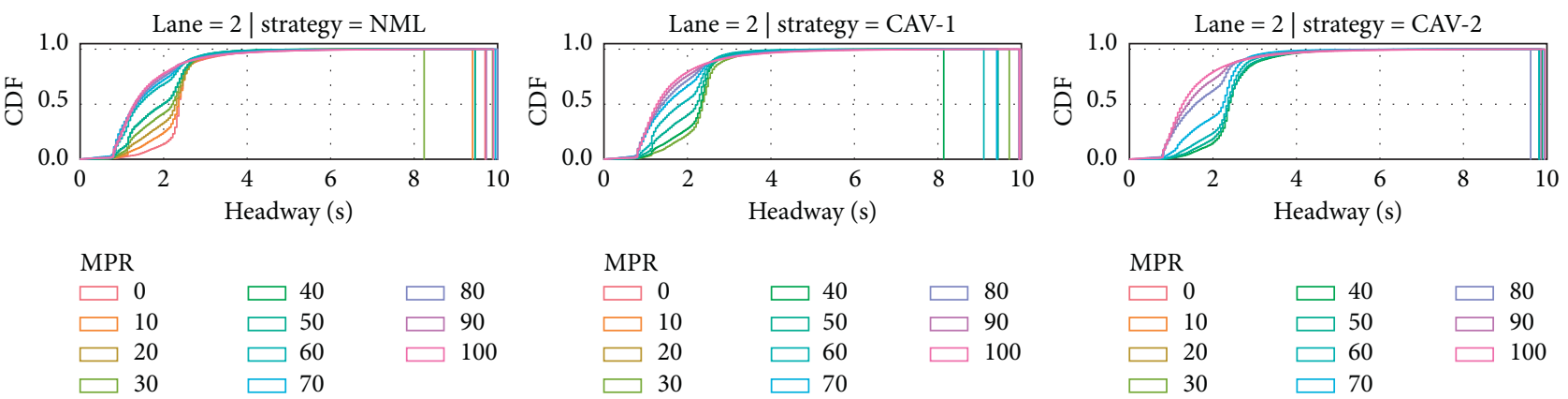

MPR

$\square 0$

$\square 10$

$\square 20$

$\square 40$

$\square 80$

$\square 30$

$\square 50$

$\square 90$

$\square 30$

$\square 70$

$\square 70$
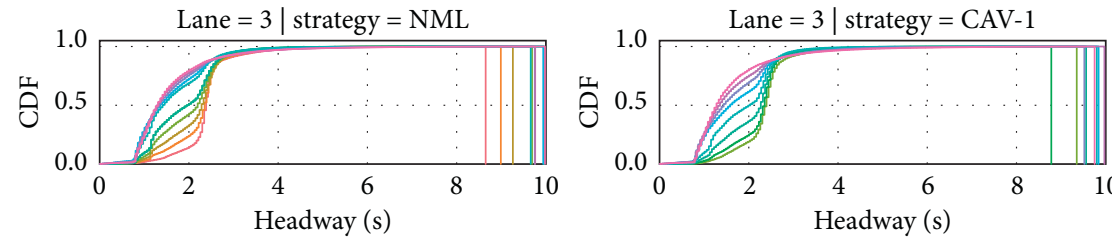

\section{MPR}

$\square 0 \quad \square 40 \quad \square 80$

$\square 10$

$\square 20$

$\square 50$

60

$\square 90$

30

$\square 70$

$\square 100$
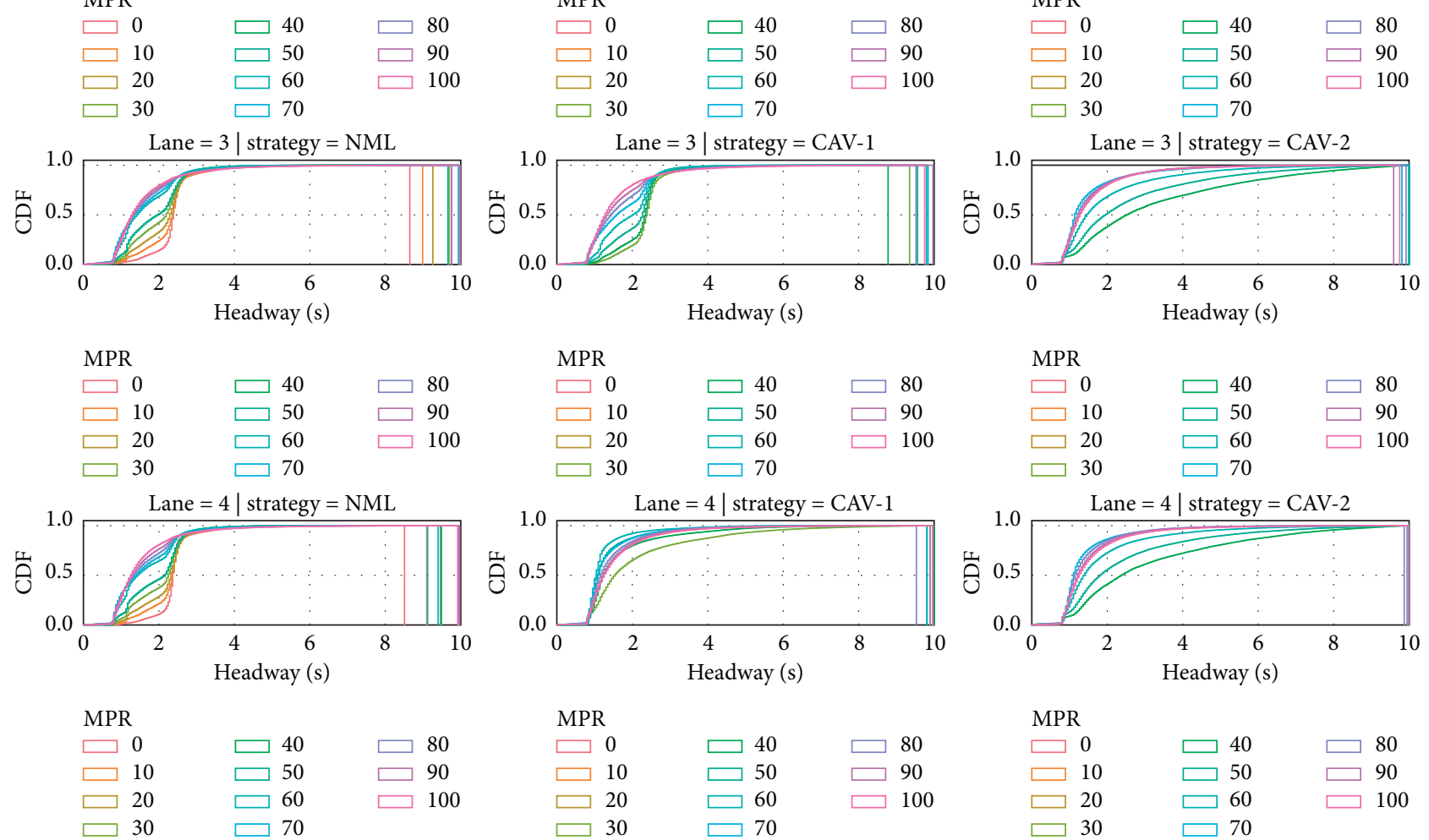

FIgURE 3: Cumulative distribution for headway distribution among travel lanes.

contrast to managed lane cases. For the CAV-2 case, the separation of the CAV lanes (the left-most and the second left-most) started to appear at $70 \%$ MPR. At full penetration $(100 \%)$, the traffic patterns are very similar, as the managed lane becomes irrelevant.

4.2. Headway Distribution. The simulation collects raw data from the data collector, an equivalent of real-world detectors (e.g., loop detectors, video cameras, and microwave sensors). By analyzing the high-resolution raw data (collected every $0.1 \mathrm{~s})$, the headway distribution in CAV lanes can be obtained. Recall that the collectors are placed in three sections of the roadway segment, as shown in Figure 1.

The cumulative probability function (CDF) curves are displayed in Figure 3. The vertical lines in the figure are the headways when $100 \%$ cumulative probability is reached. The slope of the CDF indicates the level of concentration of the samples within a distribution. In NML cases, two types of tipping points exist: the one at lower headway resulting from a high MPR and the one with higher headway observed at a low MPR (below 40\%). For CAV-1, the pattern for CDF at $30 \%$ and $40 \%$ is transformed to the pattern observed at high MPRs. With $2 \mathrm{CAV}$ lanes, the CDF increases gradually in the 


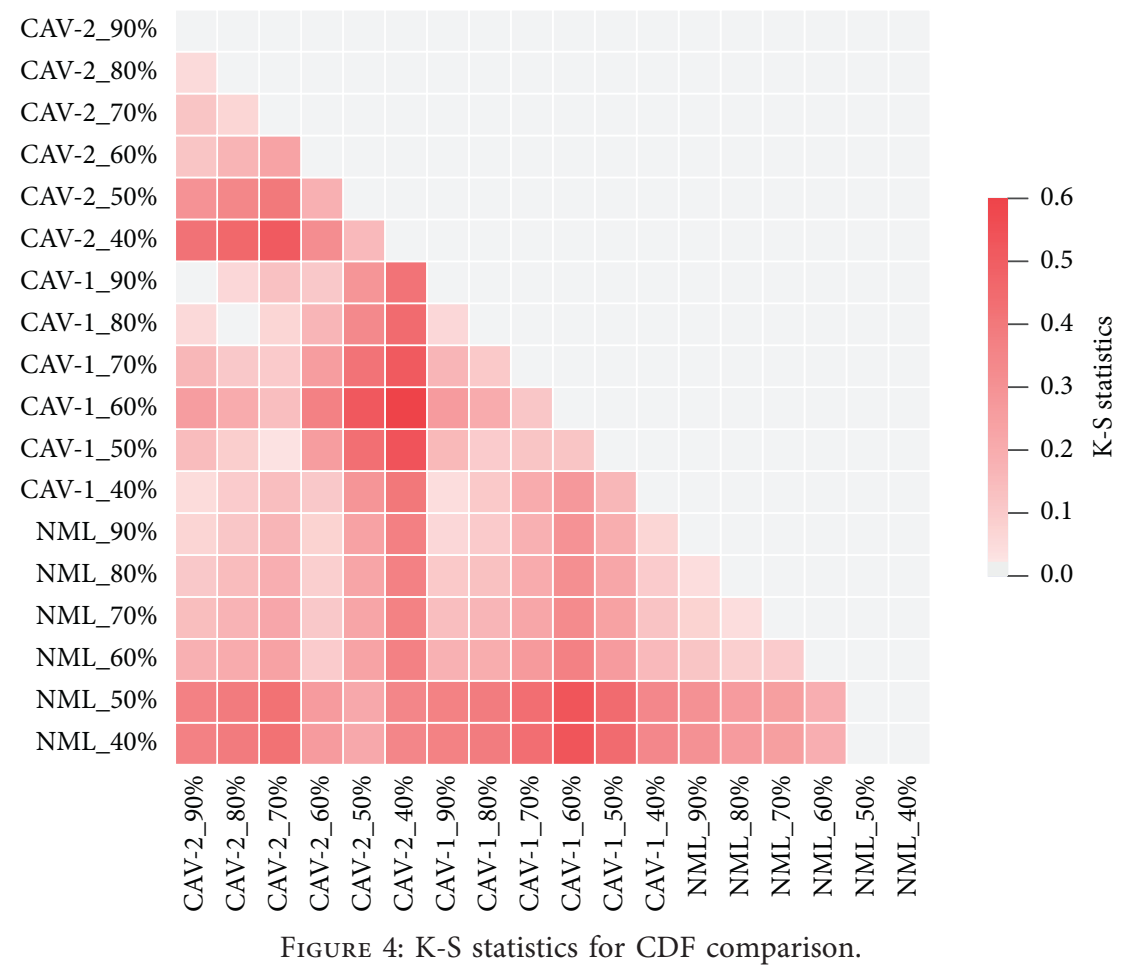

mid-range MPR (40\% to $60 \%$ ) because of undersaturation on the CAV lanes, as illustrated in the CDF on the 3rd and 4th lanes. Such undersaturation situation is alleviated when the MPR reaches $70 \%$. A similar pattern in CDFs is observed at a high MPR range (i.e., $80 \%$ to $90 \%$ ) regardless of the managed lane strategies, indicating a high concentration of samples with headway above $1 \mathrm{~s}$.

Two-sample Kolmogorov-Smirnov (K-S) test is adopted to analyze the CDFs to check whether two random samples are from the same population [68]. It is a nonparametric test where no assumption is made regarding the distribution of the variables [69]. The null hypothesis $\left(H_{0}\right)$ of the twosample K-S test is that the two sample sets are from the same continuous distribution. Nearly all the CDFs in the pairwise comparison reject the null hypothesis with a low $p$ value at the 0.05 significance level, with the exception of the comparison of $40 \%$ and $50 \%$ in NML. Figure 4 is a heatmap that shows the pairwise K-S statistics that represent the supremum of the two tested empirical CDFs. The denser the color, the higher the difference in cumulative probability between two comparing scenarios.

The average headway for HVs and CAVs in every travel lane is shown in Figure 5. The row represents the vehicle types, whereas the column represents the travel lane. Recall that the 4th lane is the left-most lane. For HVs, their averaged headway decreases as the MPR increases in CAV-1 and CAV-2 cases. While the headway also decreases in the NML case, it is at a lesser rate. When it comes to CAVs, the decreasing rate in CAV-2 is greater than that in CAV-1 or NML. The mean headway is around $4 \mathrm{~s}$ in CAV-2 case when the MPR is low or in middle range due to low lane utilization in the CAV lanes. The average headway in CAV-2 case reaches a comparable level to its counterparts at 70\% MPR, which is the deflection point. The lowest mean headway achieved among all scenarios is observed at 70\% MPR in CAV-1 case for CAVs, which corresponds to the maximum capacity with all other factors being equal. Lastly, the headway trend for CAVs remains a similar pattern across four travel lanes in the NML case, since CAVs are uniformly distributed across all lanes.

Figure 6 shows the comparison of headway distributions in the left-most lane among three managed lane scenarios under different MPRs. In the $40 \%$ to $70 \%$ MPR range, it is shown that implementing a managed lane for CAVs clearly shifts the distribution to the left-hand side, which represents smaller headways. The distributions of headway collected for either CAV-1 or CAV-2 become "narrower" (with less standard deviation), as the MPR increases from $40 \%$ to $70 \%$. The highest bin of the histogram for both CAV-1 and CAV-2 cases is $1 \mathrm{~s}-1.2 \mathrm{~s}$ when the MPR is below $50 \%$. When the MPR is higher than $50 \%$, the highest bin of the histogram shifts to $0.8 \mathrm{~s}-1 \mathrm{~s}$. In comparison, the NML case does not exhibit such a concentration pattern as the MPR increases. The result indicates that a homogeneous traffic flow comprised of only CAVs is able to realize the short headway benefits from deploying CAVs.

4.3. Fuel Consumption. The VT-Micro model [16], an individual vehicle- and operation-level emission model, is adopted to calculate the instantaneous fuel consumption rate. The inputs for the VT-Micro model are instantaneous vehicle speed and acceleration, and the output is the secondby-second fuel consumption rate, as shown in equation (5), where $\dot{x}$ is the instantaneous speed, $\ddot{x}$ is instantaneous acceleration, and $L_{i, j}^{e}$ and $M_{i, j}^{e}$ are regression coefficients for emission and fuel consumption at speed power $i$ and acceleration power $j$, respectively: 

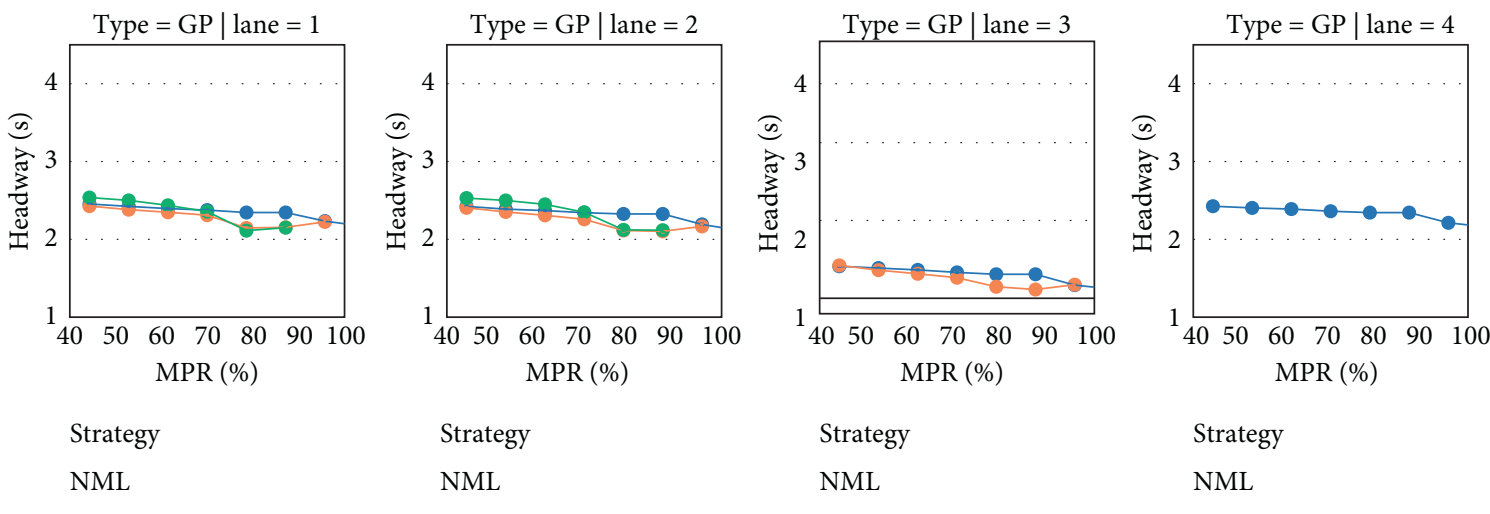

Strategy

Strategy

Strategy

CAV-1

NML

NML

NML

CAV-2

CAV-1

CAV-1

CAV-1

CAV-2

CAV-2

CAV-2

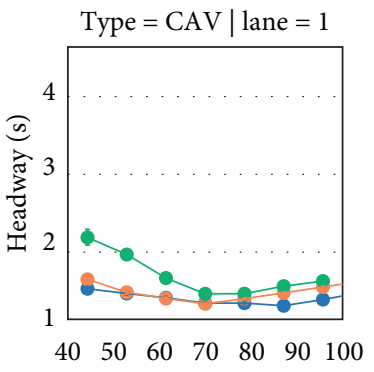

Type $=\mathrm{CAV} \mid$ lane $=2$

Type $=$ CAV $\mid$ lane $=3$

Type $=\mathrm{CAV} \mid$ lane $=4$
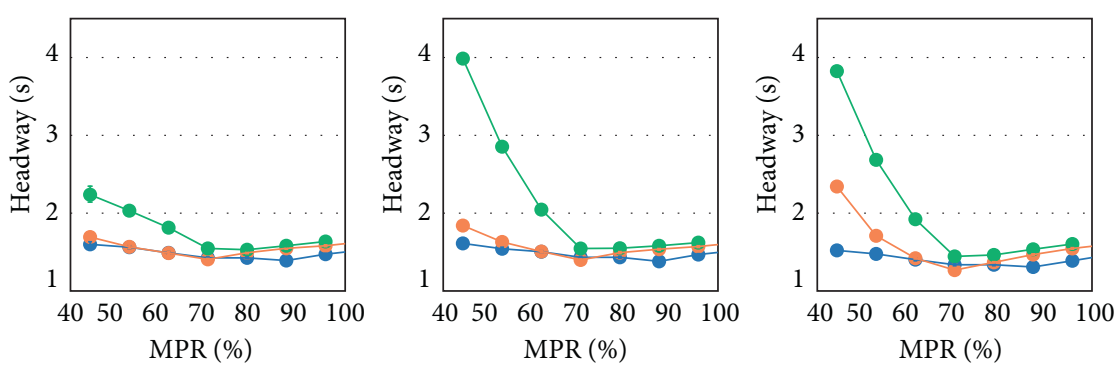

Strategy

Strategy

NML

Strategy

Strategy

NML

CAV-1

NML

NML

CAV-1

CAV-1

CAV-2

CAV-2

CAV-2

CAV-2

Figure 5: Average headway.

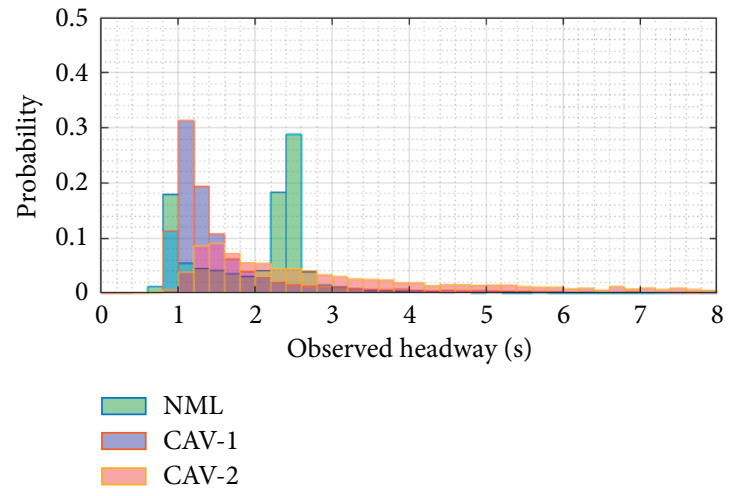

(a)

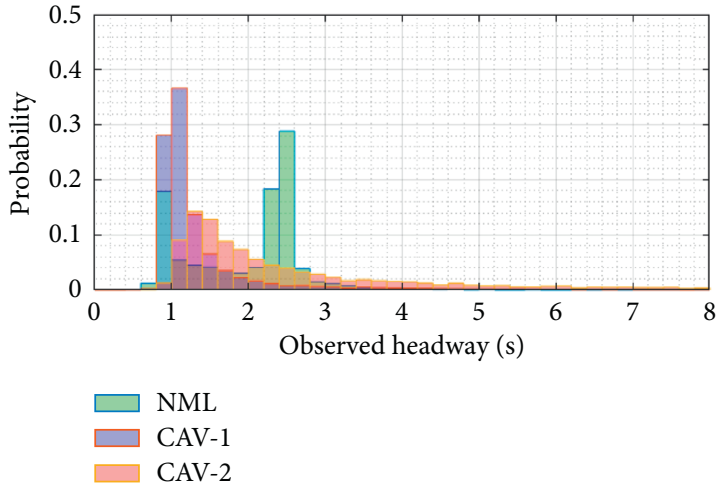

(b)

Figure 6: Continued. 

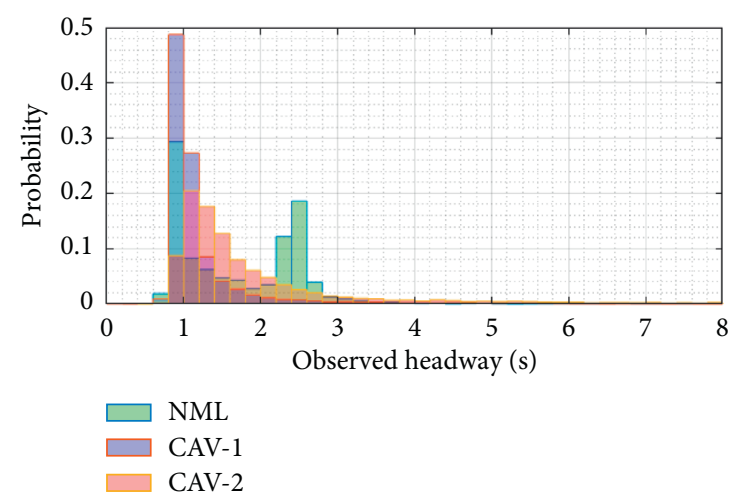

(c)

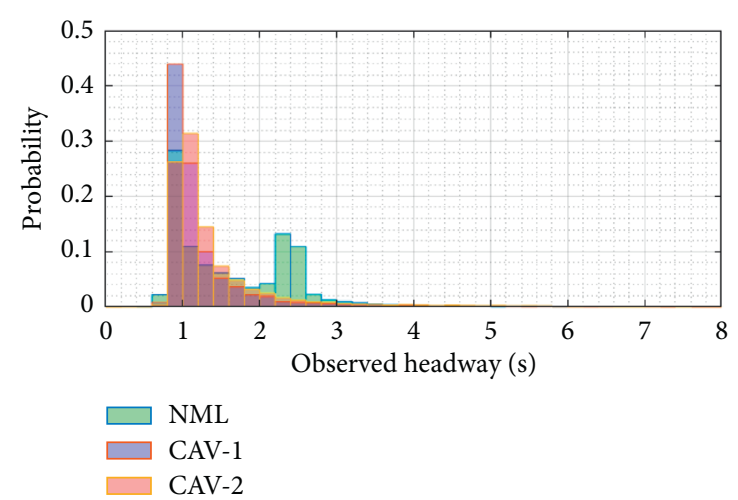

(d)

Figure 6: Headway distributions in the left-most lane. (a) 40\% MPR. (b) 50\% MPR. (c) 60\% MPR. (d) $70 \%$ MPR.

$$
f(\dot{x}, \ddot{x})= \begin{cases}\exp \left(\sum_{i=0}^{3} \sum_{j=0}^{3}\left(L_{i, j}^{e} \cdot \dot{x}^{i} \cdot \ddot{x}^{j}\right)\right), & \text { for } \ddot{x} \geq 0, \\ \exp \left(\sum_{i=0}^{3} \sum_{j=0}^{3}\left(M_{i, j}^{e} \cdot \dot{x}^{i} \cdot \ddot{x}^{j}\right)\right), & \text { for } \ddot{x}\end{cases}
$$

The vehicle data was derived from the raw data from the detectors in three locations marked in Figure 1. The result for the fuel consumption is plotted in Figure 7, which shows two distinctive patterns for the GPLs and the managed lane. The concentration of fuel consumption is within $5 \mathrm{ml} / \mathrm{s}$ to $10 \mathrm{ml} / \mathrm{s}$ for lanes that allow HV operation (i.e., mixed traffic), when the MPR for CAVs is equal to or less than $60 \%$. When the MPR rises to above $60 \%$, the instantaneous fuel consumption shifts to lower values with a "narrower" slope: higher concentration between $5 \mathrm{ml} / \mathrm{s}$ and $7 \mathrm{ml} / \mathrm{s}$.

We then isolate the CDF curve for both CAVs and HVs, when they operate on the left-most lane under homogeneous flow condition. More specifically, the separated CDF curves represent the observations of HVs from the 0\% MPR in NML case and the observations for CAVs from the $100 \%$ MPR for CAV-1 case. The CDF curves in Figure 7 exhibit two different patterns for CAVs and HVs. The former with $60 \%$ of the observations fall below $4 \mathrm{ml} / \mathrm{s}$, whereas the latter with $60 \%$ of the observations fall below $12 \mathrm{ml} / \mathrm{s}$ with a wider spread. The wider spread for HVs is probably caused by the stochastic nature of human drivers (which is simulated by the Wiedemann model). Hence, the mixed traffic condition is comprised of two competing flows that excrete their influence.

In the GPLs, the MPR plays a role as an indicator for the dominance of each traffic flow. The higher the MPR is, the closer the CDF curves approach the pattern of managed lane that is used by CAV exclusively. In the managed lane, the CAV traffic is the sole dominating traffic. Therefore, the fuel consumption curve exhibits only CAV traffic characteristics, regardless of the MPR. We include the fuel consumption rate CDF curves for HVs and CAVs in Appendix C, Figures 8 and 9: both figures demonstrate the shift towards CAV fuel consumption CDF pattern as the MPR grows. The difference in fuel consumption between two types of homogeneous flow can be found in Figure 10 in Appendix.

4.4. Wireless Communication. Figure 11(a) shows the maximum and the average density for instances of $\mathrm{V} 2 \mathrm{~V}$ communication among three managed lane policies. Recall that the DSRC communication model only deals with the physical layer. While the transmission density increases as the MPR increases, the maximum density in NML is higher than CAV-1 and CAV-2, because the CAV platoons were broken down by certain HVs, which are susceptible to shockwaves. As such, the traffic flow is compressed, producing a higher traffic density and thus higher transmission density. With the aid of CAV lane, the communication density is thus maintained at a lower level. In a CAV lane, the CAVs distribute longitudinally on the managed lane. The $\mathrm{NML}$, in comparison with two managed lane cases, is more likely to generate pockets of traffic with CAVs across multiple lanes, which could result in localized higher transmission activity.

The probability of successful reception of BSM from a leading vehicle to a subject vehicle is shown in Figure 11(b). The probability curves under CAV-1 and CAV-2 scenarios are in close proximity to each other and they are showing the same trend. The maximum difference between these two curves is 0.04 at $90 \%$ MPR. The probability of successful communication of NML at high MPR range (60\% to $90 \%$ ) is consistently lower than those of CAV-1 and CAV-2. This is caused by the compression of traffic flow by localized shockwaves. There is an overall decreasing trend of the probability as the MPR increases but there still remains a successful rate of $94 \%$ and above.

4.5. Network Performance. The measures used in this section gauge the overall performance of the simulation network at an aggregated level. The throughput represents the total number of vehicles that have arrived at their destinations, 

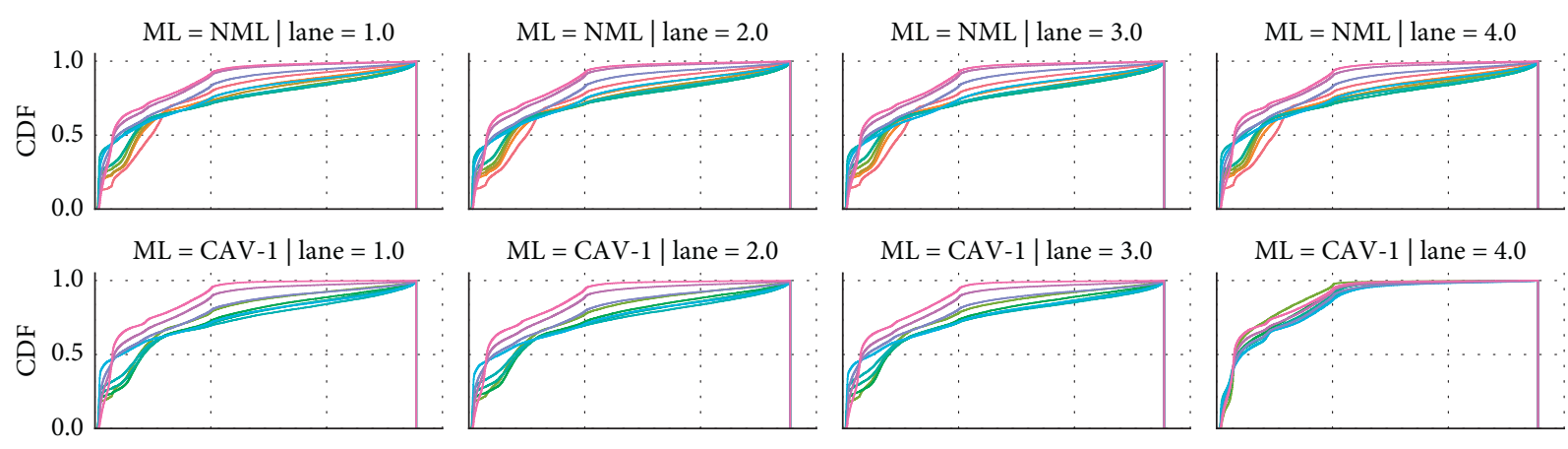

$\mathrm{ML}=\mathrm{CAV}-1 \mid$ lane $=4.0$
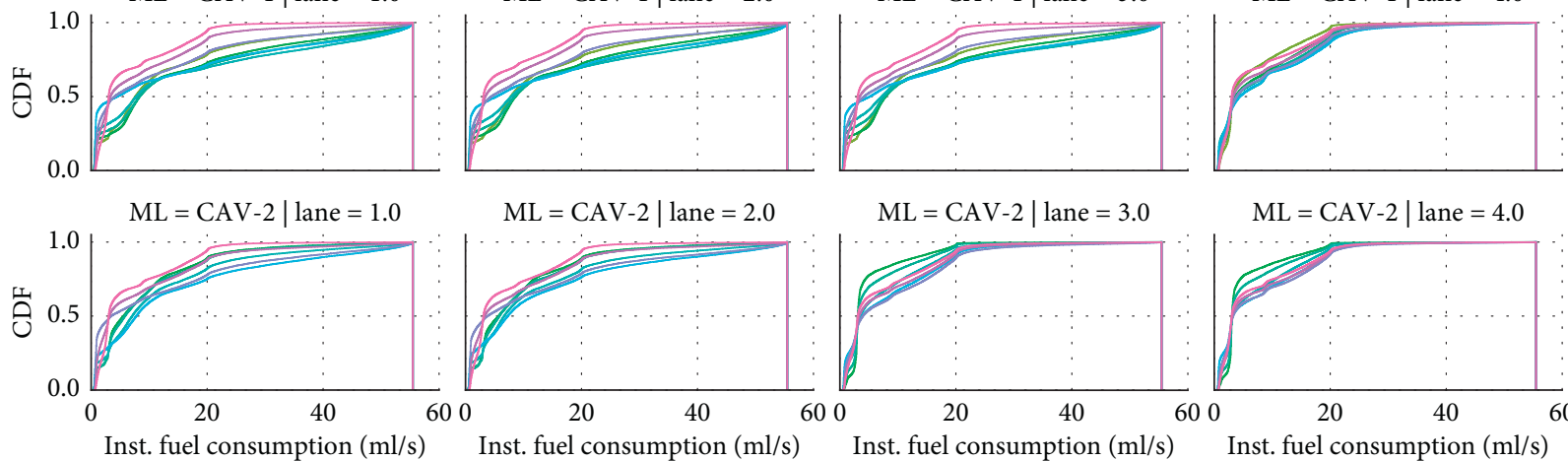

$\mathrm{ML}=\mathrm{CAV}-2 \mid$ lane $=4.0$

MPR
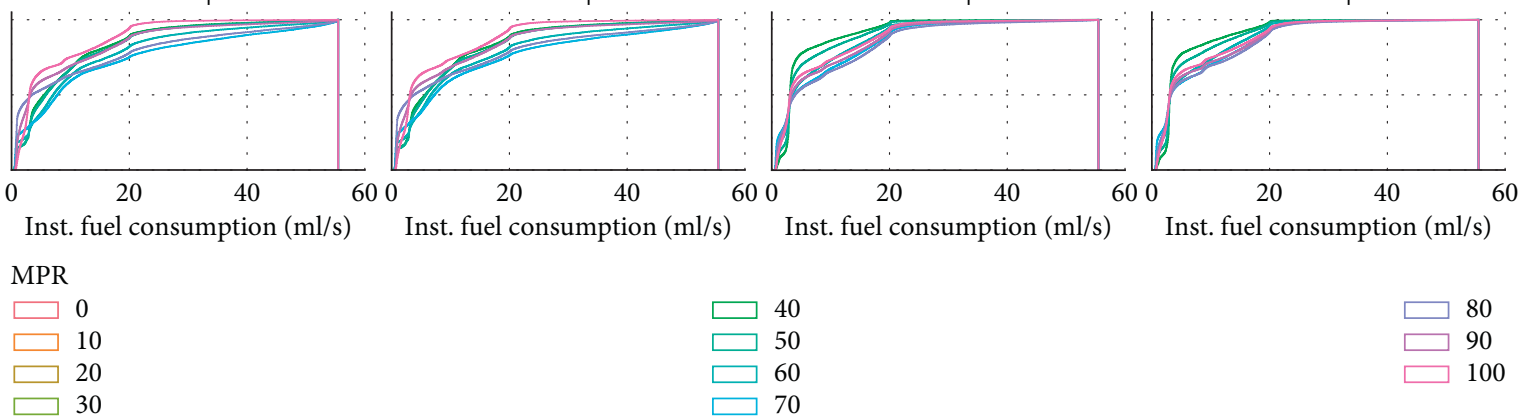

FIgURE 7: Instantaneous fuel consumption for all vehicles.
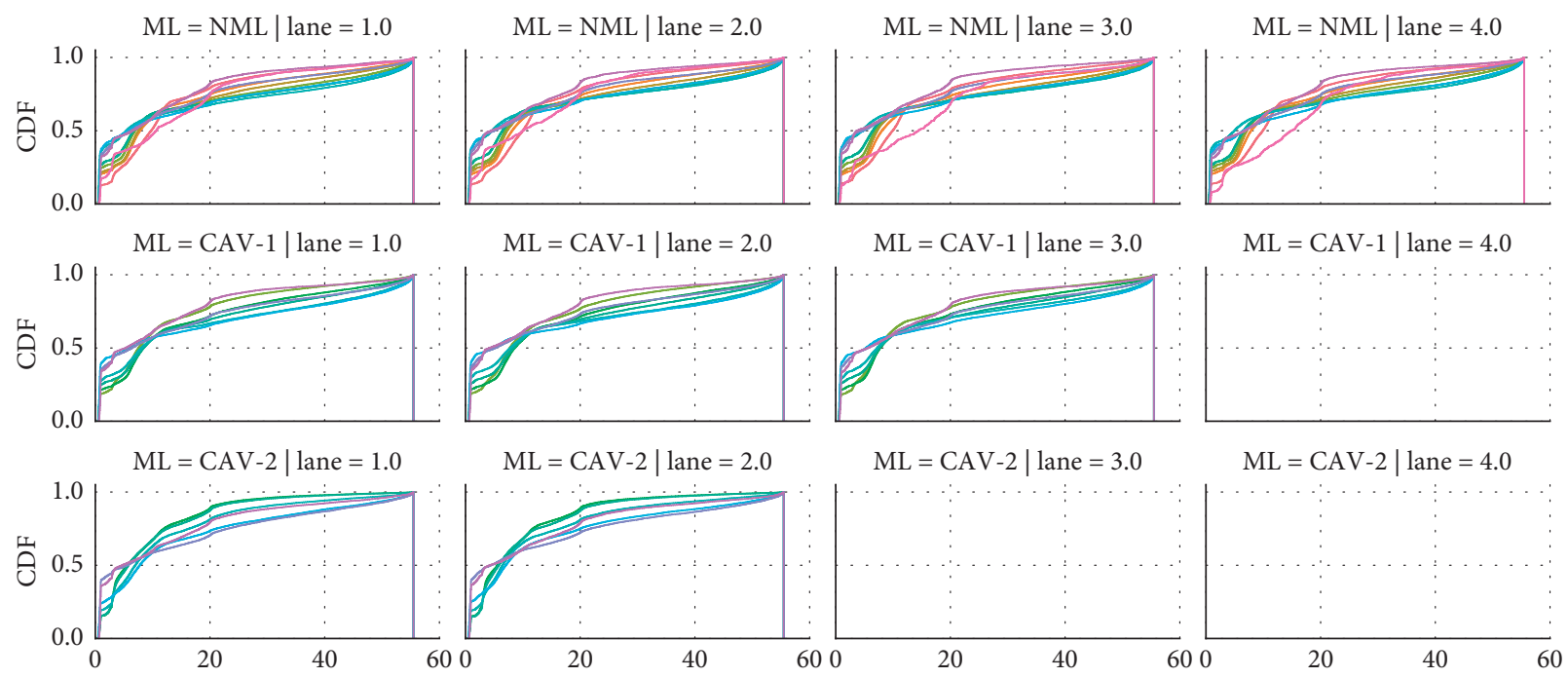

Inst. fuel consumption $(\mathrm{ml} / \mathrm{s})$
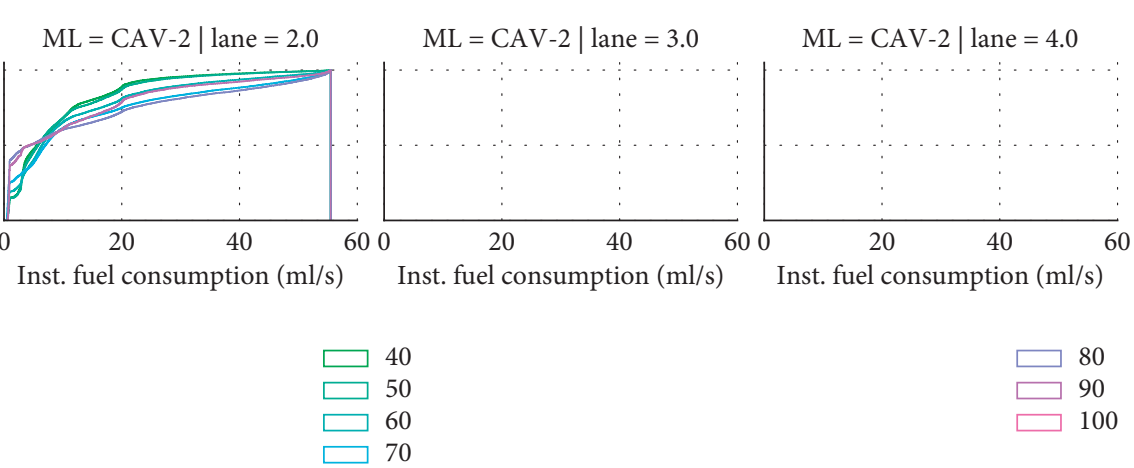

MPR

$$
\begin{aligned}
& \square 0 \\
& \square \quad 10 \\
& \square \quad 20 \\
& \square \quad 30
\end{aligned}
$$

FIgURE 8: Instantaneous fuel consumption for HVs.

shown in Figure 12. As mentioned before, the network was configured with a higher than usual demand. With a $10,000 \mathrm{vph}$ demand for a four-lane highway, the network was only able to process $6500 \mathrm{vph}$ in the absence of CAVs. Under the NML scenario, as the MPR of CAVs increases, so does the network throughput. The throughput reaches approximately $8000 \mathrm{vph}$ with $40 \%$ and $50 \%$ MPRs. However, at $60 \%$ MPR, the network throughput is boosted again and remains at the same level at $9600 \mathrm{vph}$ when the MPR is above $70 \%$. The throughput in CAV-1 case begins to outperform the NML case at MPR 50\% and keeps increasing to $9700 \mathrm{vph}$ at $70 \% \mathrm{MPR}$, where the throughput starts leveling in spite of 

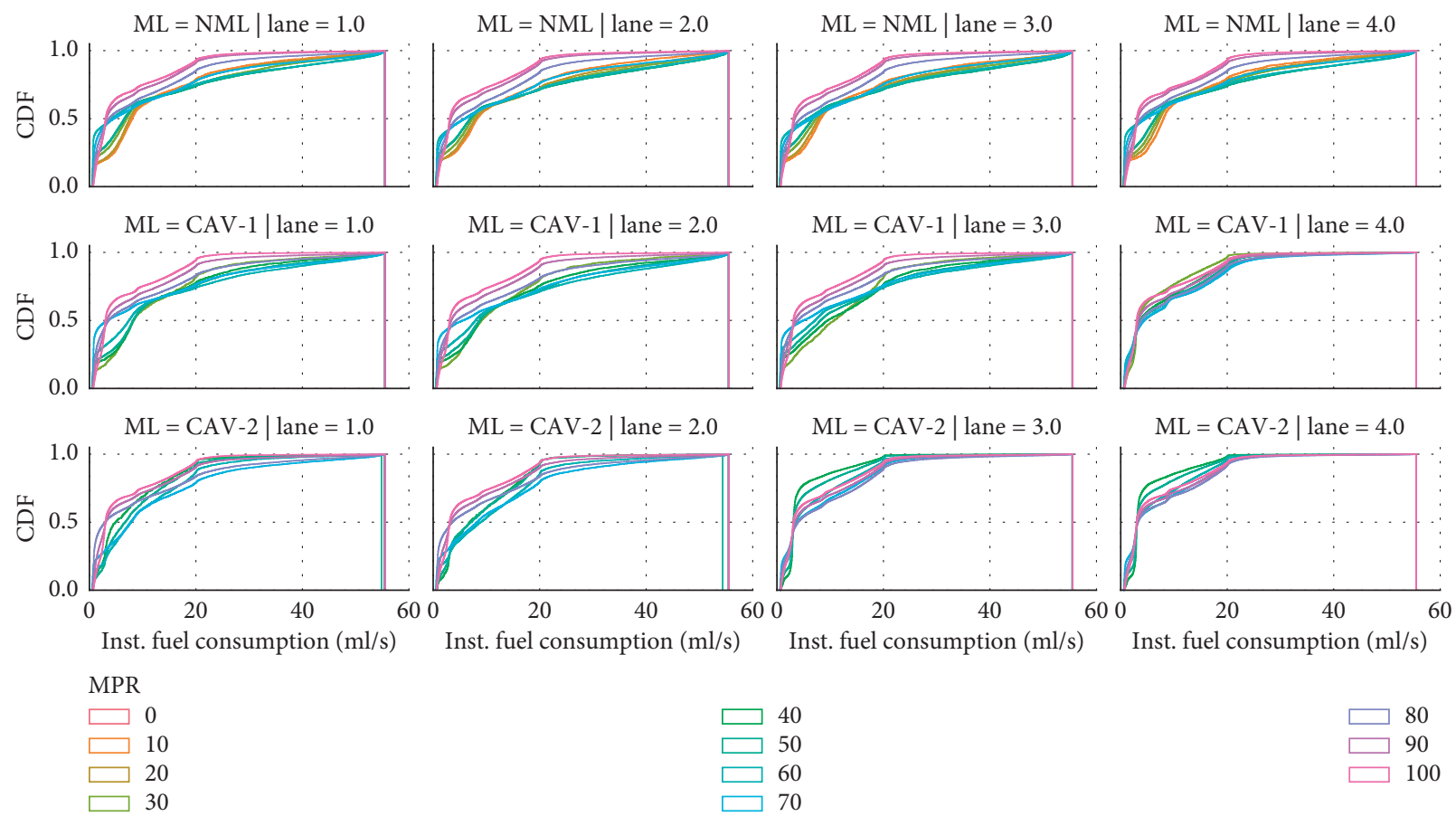

FIgURE 9: Instantaneous fuel consumption for CAVs.

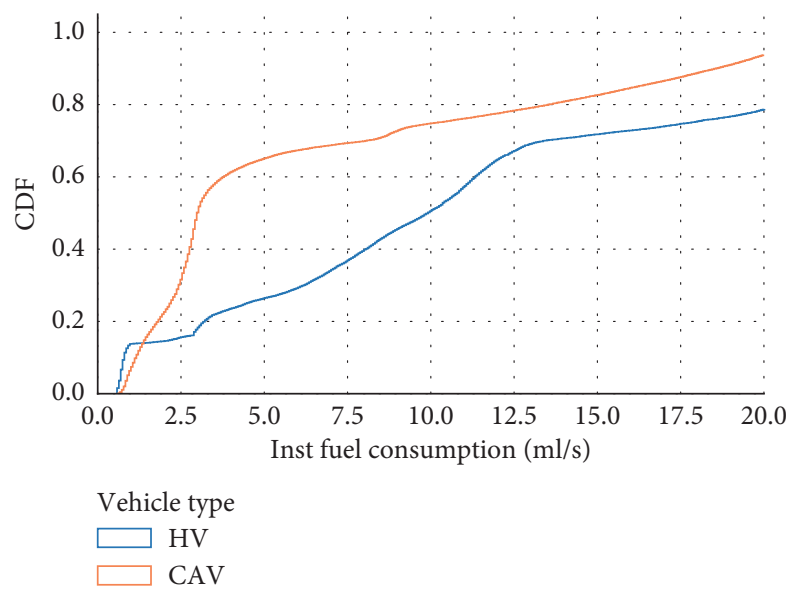

FIGURE 10: Instantaneous fuel consumption curve for homogeneous flow.

the increase in MPR. For the CAV-2 policy, the system throughput only reaches the same level of the two counterparts at 70\% MPR due to underutilization of CAV lanes with low MRPs.

The average delay experienced by vehicles (plotted in Figure 13(a)) within the network is calculated by dividing the total delay by the sum of the vehicles within the network and the vehicles that have exited the network. For three strategies, the average delay starts to decrease as the throughput levels off: at $60 \%$ for NML and CAV-1 and at $70 \%$ for CAV-2. Such seemingly counter-intuitive phenomena could be explained by taking into account the average speed, which is shown in Figure 13(b): when the throughput is in a graduate increase as the MPR goes until $60 \%$, the average speed exhibits a decreasing trend, which is in an inverse relationship with vehicle delay. This trend is in agreement with the speed-flow fundamental diagram. 


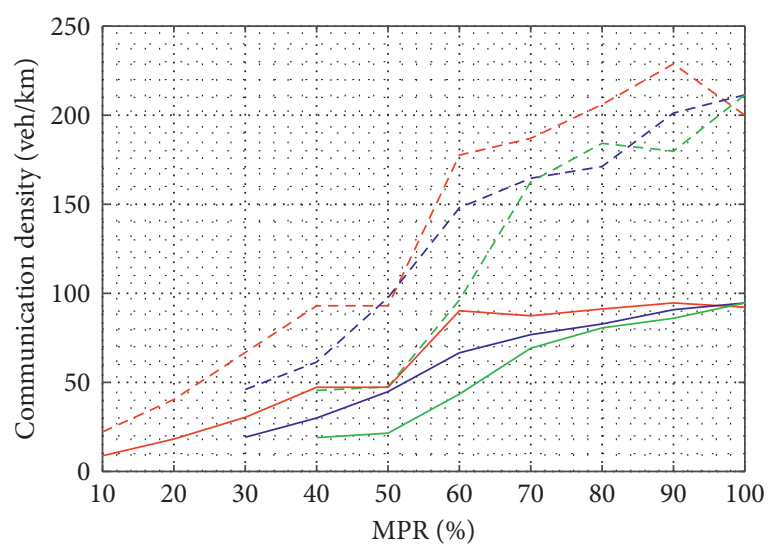

ML policy

- - Max. density NML _— Avg. density NML

- - - Max. density CAV-1 — Avg. density CAV-1

- - - Max. density CAV-2 — Avg. density CAV-2

(a)

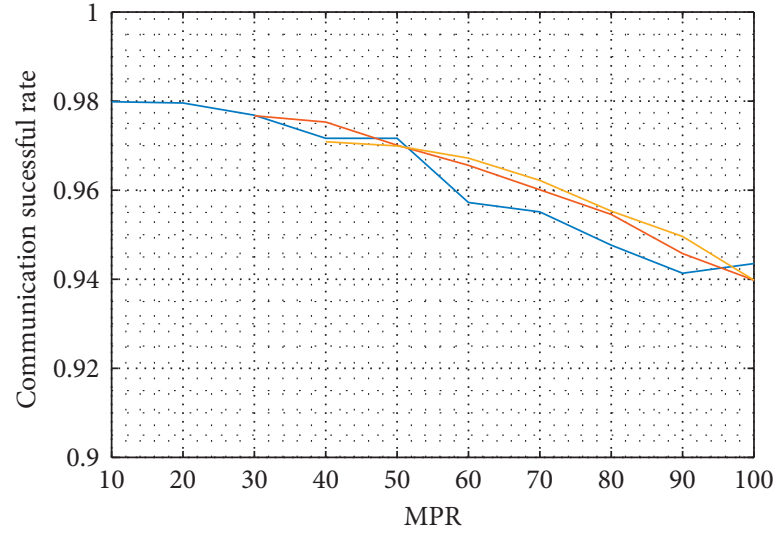

ML policy

$-\mathrm{NML}$

$-\mathrm{CAV}-1$

CAV-2

FIGURE 11: V2V communication performance measure. (a) Vehicle density. (b) Packet perception rate.
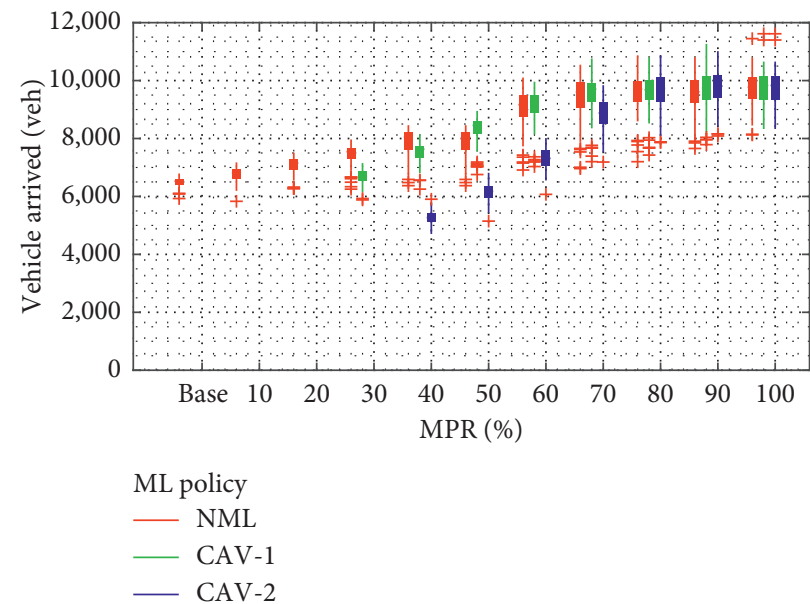

FiguRe 12: Network throughput.

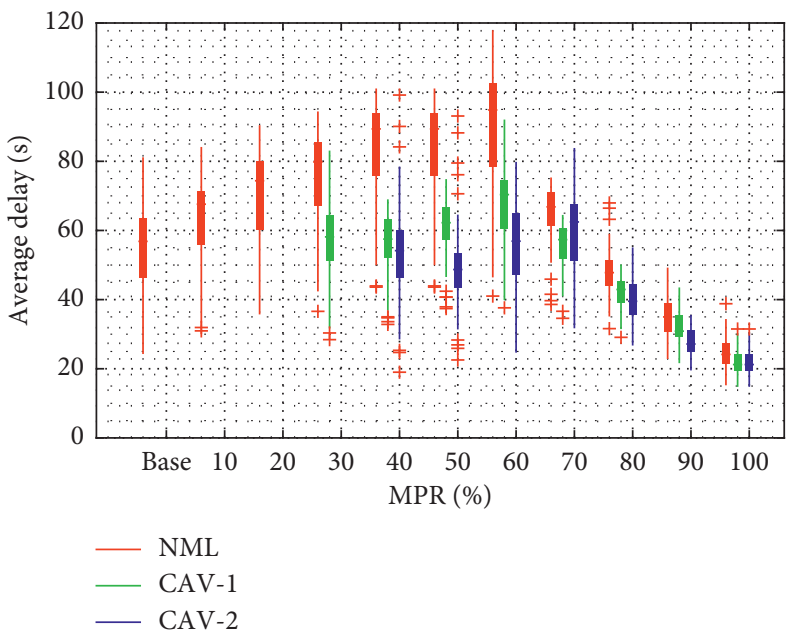

(a)

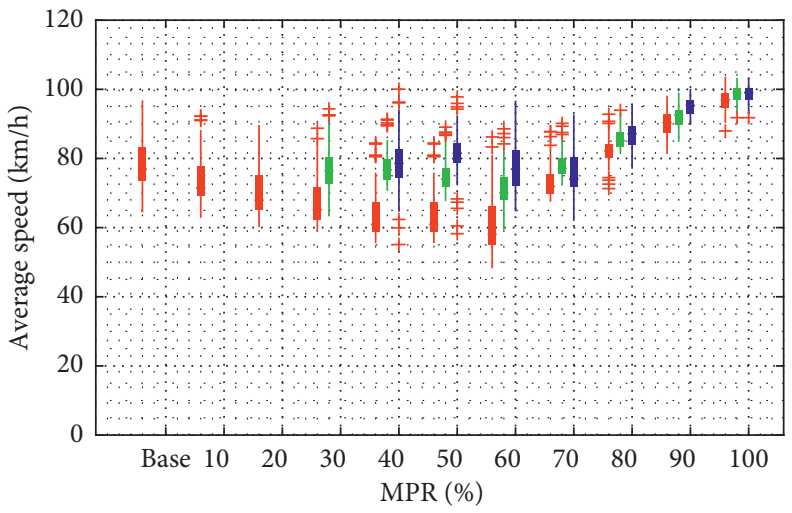

ML policy
NML
CAV-1
CAV-2

(b) 


\begin{tabular}{|c|c|c|c|c|c|}
\hline & \multicolumn{5}{|c|}{$(\mathrm{j}, \mathrm{k})$} \\
\hline & $(0,0)$ & $(1,0)$ & $(2,0)$ & $(3,0)$ & $(4,0)$ \\
\hline$\overline{h_{1}^{(j, k)}}$ & 0.0209865 & $-9.66304 e-07$ & $-1.72786 e-11$ & $5.09506 e-17$ & $-7.91921 e-23$ \\
\hline$h_{2}^{(j, k)}$ & 2.24743 & $7.84884 e-07$ & $2.28533 e-10$ & $-5.89802 e-16$ & $3.55262 e-22$ \\
\hline$h_{3}^{(j, k)}$ & 2.56426 & $2.82287 e-05$ & $-7.09939 e-10$ & $1.34371 e-15$ & $-3.01956 e-22$ \\
\hline$h_{4}^{(j, k)}$ & 2.41146 & $-9.32859 e 05$ & $6.77403 e-10$ & $-9.64188 e-16$ & $3.69652 e-23$ \\
\hline & $(3,1)$ & $(2,1)$ & $(2,2)$ & $(1,1)$ & $(1,2)$ \\
\hline$\overline{h_{1}^{(j, k)}}$ & $3.16577 e-20$ & $2.13587 e-14$ & $05716 e-17$ & $4.00928 e-09$ & $-1.88707 e-11$ \\
\hline$h_{2}^{(j, k)}$ & $4.07120 e-19$ & $-2.66510 e-13$ & $8.64273 e-17$ & $-7.31274 e-08$ & $2.98549 e-10$ \\
\hline$h_{3}^{2(j, k)}$ & $-1.85451 e-18$ & $1.02847 e-12$ & $1.80250 e-16$ & $1.56259 e-07$ & $-8.50944 e-10$ \\
\hline$h_{4}^{(j, k)}$ & $1.85043 e-18$ & $-1.13894 e-16$ & $-4.05333 e-16$ & $-2.56738 e-08$ & $6.24415 e-10$ \\
\hline & $(1,3)$ & $(0,1)$ & $(0,2)$ & $(0,3)$ & $(0,4)$ \\
\hline$\overline{h_{1}^{(j, k)}}$ & $3.25406 e-14$ & 0.000418109 & $-4.30875 e-06$ & $1.00775 e-08$ & $-7.32254 e-12$ \\
\hline$h_{2}^{(j, k)}$ & $-3.24982 e-13$ & 0.00498750 & $-7.22232 e-06$ & $1.69755 e-08$ & $-2.94381 e-11$ \\
\hline$h_{3}^{(j, k)}$ & $7.59094 e-13$ & -0.0227008 & $7.50391 e-05$ & $-1.81469 e-07$ & $2.02182 e-10$ \\
\hline$h_{4}^{(j, k)}$ & $-3.57571 e-13$ & 0.0191490 & $-6.92678 e-07$ & $1.79917 e-07$ & $-2.07263 e-10$ \\
\hline
\end{tabular}

(a)
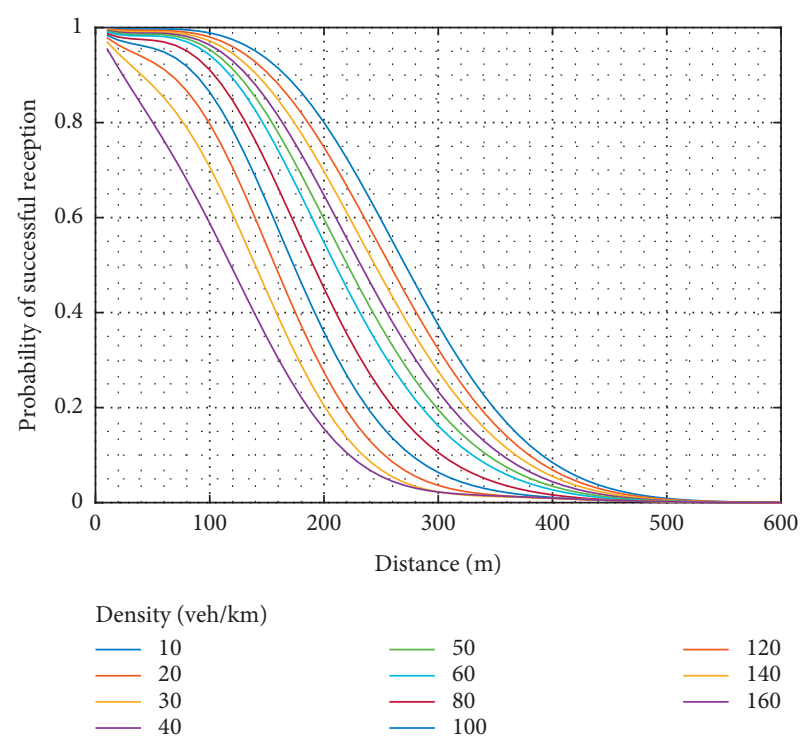

(b)

FIGURE 14: DSRC model coefficients and PDF. (a) Coefficient $h_{i}^{(j, k)}$ in equation (4). (b) PDF of successful reception (300 m power range).

\section{Discussion and Conclusions}

In this section, we highlight the findings from the previous section and discuss the study in a boarder context.

5.1. Summary. The analysis results indicate that the introduction of CAV could increase the throughput of the overall system, even when no managed lane policy is in place. The congestion region in the speed-flow diagram disappears as the MPR of the CAVs increases. This is an indication of the improvement of roadway capacity owing to CAVs, which is consistent with the findings of previous studies. More importantly, the congestion region first disappears in the CAV lane in CAV-1 case, illustrating that the homogeneity of CAV traffic results in a more stable traffic flow with a high throughput. A CAV lane, with an MPR as low as $40 \%$, is able to accommodate more traffic compared to a GP lane and it helps to alleviate the overall congestion of the network. The average vehicle delay exhibits a decreasing trend, even after the network throughput levels after $70 \%$ MPR. This is an indicator that the network is able to carry more traffic than the high demand specified in Figure 1.

The individual headways among consecutive vehicles are measured for each lane. From the headway distribution, one can measure not only the compactness of the traffic but also the stability of the traffic flow. Both HVs and CAVs have a predominate headway as shown in Table 4 . In a heterogeneous traffic flow, two spikes with different tipping points can be observed in the headway CDF curve. Each segment indicates a high concentration for the headway samples. One is for the following headway samples observed on HVs, and the other is for the headway samples for CAVs. With traffic homogeneity on the CAV lane, there is only one spike on the CDF curves. The magnitude of the spike also depends on the lane occupancy, as evidenced by the comparison of CAV-1 and CAV-2 at the same MPR. The two-spike pattern remains even at high-range MPR (i.e., 60-80\%) in the absence of CAV lane (the NML case).

The VT-Micro model, which produces instantaneous fuel consumption for individual vehicles, was employed to estimate the environmental impact of the CAV lane. The vehicle speed and acceleration were collected as inputs and the relative fuel consumption, instead of the absolute one, was examined. Again, distinct patterns for a GPL and a CAV lane were observed. The average instantaneous fuel consumption for CAV lane has a narrower distribution.

Lastly, the DSRC communication was measured using an analytical communication model that is derived from a package-level network simulator. It simulates the physical layer of the DSRC communication that is an integral element of CAVs. We found a lower communication density in CAV lane, as the CAVs were more evenly distributed longitudinally. A lower communication density indicates a less congested communication channel, which increases the performance of the $\mathrm{V} 2 \mathrm{~V}$ communication. Compared to CAV-1 and CAV-2 scenarios, it is more likely under NML scenario to generate pockets of traffic with CAVs across multiple lanes, which could introduce higher localized transmission activity and increase the loss of BSM packets.

The overall results show that a single CAV lane in a fourlane highway network is able to provide the necessary technical accommodation efficiently in the mixed traffic conditions with a wide range of MPR. A CAV dedicated lane is helpful to guarantee the benefits of CAVs, as it creates a homogeneous 
CAV flow. Implementing two CAV lanes, however, may adversely affect the overall traffic, especially when the MPR of CAV does not warrant an additional CAV lane.

5.2. Limitations. While the paper demonstrates the benefits of managed lane for CAV at lane level and vehicle level, we should note that there are limitations in this study and the benefits are realized in a controlled environment under certain assumptions. First of all, although the Wiedemann model is behaviorally sound and has been adopted by numerous researchers for simulating human drivers, the complexity of a human driver under dynamic traffic conditions is difficult, if possible at all, to be captured by simulation models. In addition, the behavioral adaptation for human drivers in the presence of CAV is not known yet, due to the lack of empirical evidence in the public domain. Preliminary results revealed that a smaller time headway was adopted by a HV when driving along side closed platooned CAVs [70]. Note that the Wiedemann driver behavior parameters were calibrated using field data where CAVs have not been deployed on the roadway yet. The calibrated parameters represent a subset of the driving population, and they may not be directly transferable to other driving conditions or demographics. The E-IDM, while being widely adopted, does not contain the multianticipative car-following feature, which has been promoted as one of the crucial features enabled by V2V communication. Therefore, the performance of the CAVs is expected to be more conservative. Like many existing CAV car-following models, the E-IDM does not factor the aspects of human factor that is anticipated to be more pronounced in the lower levels of automation.

In addition, there are several salient issues regarding the low-level automation and its modeling as well. For a CAV, the drivers' acceptance of short following headway (e.g., $0.6 \mathrm{~s}$ ) is still an open question [24], given that the short following headway is technologically attainable. It is reasonable to expect that the acceptance of extremely short headway would be low initially, although it will gradually increase as CAV penetration increases. The pace of adaption, though, is largely depending on the level of confidence to the ADS from human drivers. The level of compliance from drivers (in the absence of automation) is also an important factor in harnessing rich information brought by the connectivity. The layer of driver stochasticity in reacting to traffic information remains. In the extreme case, a complete disregard of useful information could negate the benefits of connectivity.

Another crucial issue is the transition of control from the ADS back to the human driver. As per the definition of vehicle automation by the SAE, the level 3 automation (and below) requires a fallback receptive driver when the ADS exits its designed operational domain. As studies have shown, such fallback process is way more complicated than merely retaking the steering wheel. First, a driver needs to regain situational awareness of the traffic environment from the disengagement of driving. The surge in cognitive demand during the initial period of reengaging in driving tasks could result in deterioration in driver's performance (e.g., increased reaction time and inadequate situational awareness). This aspect rarely exists in current CAV models, and much likely it will require an endogenous cognitive model that is able to take into account the driving task demand and the cognitive capacity of human drivers [53]. Therefore, the human-machine interfacing is seldom captured in current simulation model, including the one used in this study.

5.3. Future Research. The future research would focus on relaxing the assumptions in this study. The first direction is the CAV behavior modeling. Researchers have recently started the incorporation of human factor aspect, such as an extension module in IDM to model driver's responses to advanced traffic information [57], and an explicit human cognitive model for the transition of control [53]. Such developments offer a great opportunity to introduce human factor in a mixed traffic flow in the future. Second, the innermost lane is generally assigned as the managed lane in current practices, which requires eligible users to merge to access the managed lane and induces additional demand of lane changing. The access plan (e.g., ingress and egress points of the managed lane, eligibility) requires further study to minimize the negative impacts caused by induced weaving activity. A cost-benefit analysis may also be warranted for comparing managed lane strategies with other emerging technologies, such as vehicle awareness device (VAD), for the near-term deployment of CAV. Some researchers have started the exploration of right-most managed lane in USA [65]. Lastly, the characteristics of mixed traffic flow that is anticipated in the near-term deployment of CAV need further exploration. In particular, the impact of CAVs at individual trajectory level by analyzing high-resolution vehicle trajectory data requires further exploration (Appendix A).

\section{Appendix}

\section{A. List of Abbreviations}

The list of abbreviation used is provided in Table 1.

\section{B. Coefficients for the Wireless Communication Model}

The coefficients obtained from the polynomial function $h_{i}(\xi, \varphi)$ are shown in Figure 14(a). It is worth stressing that even seemingly negligible values, if omitted, could result in deviation in the probability of reception from $8 \%$ to $100 \%$ [63]. The probability distribution curves for transmission scenarios are shown in 14(a).

\section{Instantaneous Fuel Consumption for HV and CAV}

Instantaneous fuel consumption for $\mathrm{HV}$ and CAV is shown in Figures 8 and 9. The instantaneous fuel consumption curve for homogeneous flow is shown in Figure 10. 


\section{Data Availability}

Some or all data, models, or codes that support the findings of this study are available from the corresponding author upon reasonable request.

\section{Conflicts of Interest}

The authors declare that there are no conflicts of interest regarding the publication of this paper.

\section{Acknowledgments}

This work was supported in part by the National Science Foundation under Grant No. CMMI-1844238.

\section{References}

[1] C.-Y. Chan, "Advancements, prospects, and impacts of automated driving systems," International Journal of Transportation Science and Technology, vol. 6, no. 3, pp. 208-216, 2017.

[2] Managed Lanes: A Primer, Technical Report, 2008.

[3] N. Kalra and S. M. Paddock, "Driving to safety: how many miles of driving would it take to demonstrate autonomous vehicle reliability?" Transportation Research Part A: Policy and Practice, vol. 94, pp. 182-193, 2016.

[4] S. Smith, J. Bellone, S. Bransfield et al., "Benefits estimation framework for automated vehicle operations," United States Department of Transportation, Intelligent Transportation, Technical Report, 2015.

[5] O. Hussain, A. Ghiasi, and X. Li, "Freeway lane management approach in mixed traffic environment with connected autonomous vehicles," 2016, http://arxiv.org/abs/1609.02946.

[6] R. Wang, Y. Li, and D. B. Work, "Comparing traffic state estimators for mixed human and automated traffic flows," Transportation Research Part C: Emerging Technologies, vol. 78, pp. 95-110, 2017.

[7] B. Van Arem, C. J. G. Van Driel, and R. Visser, "The impact of cooperative adaptive cruise control on traffic-flow characteristics," IEEE Transactions on Intelligent Transportation Systems, vol. 7, no. 4, pp. 429-436, 2006.

[8] S. E. Shladover, D. Su, and X.-Y. Lu, "Impacts of cooperative adaptive cruise control on freeway traffic flow," Transportation Research Record: Journal of the Transportation Research Board, vol. 2324, no. 1, pp. 63-70, 2012.

[9] R. W. Hall and H. . J. Tsao, "Capacity of automated highway systems: merging efficiency," in Proceedings of the 1997 American Control Conference (Cat. No. 97CH36041), pp. 2046-2050, Albuquerque, New Mexico, 1997.

[10] G. Arnaout and J.-P. Arnaout, "Exploring the effects of cooperative adaptive cruise control on highway traffic flow using microscopic traffic simulation," Transportation Planning and Technology, 2014.

[11] P. Songchitruksa, A. Bibeka, L. I. Lin, Y. Zhang et al., "Incorporating driver behaviors into connected and automated vehicle simulation," Center for Advancing Transportation Leadership and Safety (ATLAS Center), Technical Report, 2016.

[12] J. Lee, J. Bared, and B. Park, "Mobility impacts of Cooperative Adaptive Cruise Control (CACC) under mixed traffic conditions," in Proceedings of the 93rd Annual Meeting of the Transportation Research Board, Washington, DC, USA, January 2014.
[13] H. Liu, S. E. Shladover, X.-Y. Lu, and X. Kan, "Freeway vehicle fuel efficiency improvement via cooperative adaptive cruise control," Journal of Intelligent Transportation Systems, pp. 113, 2020.

[14] H. Liu, X. Kan, S. E. Shladover, X.-Y. Lu, and R. E. Ferlis, "Modeling impacts of cooperative adaptive cruise control on mixed traffic flow in multi-lane freeway facilities," Transportation Research Part C: Emerging Technologies, vol. 95, pp. 261-279, 2018.

[15] F. An, M. Barth, J. Norbeck, and M. Ross, "Development of comprehensive modal emissions model: operating under hotstabilized conditions," Transportation Research Record: Journal of the Transportation Research Board, vol. 1587, no. 1, pp. 52-62, 1997.

[16] H. Rakha, K. Ahn, and A. Trani, "Development of VT-micro model for estimating hot stabilized light duty vehicle and truck emissions," Transportation Research Part D: Transport and Environment, vol. 9, pp. 49-74, 2004.

[17] A. Brooker, J. Gonder, L. Wang, E. Wood, S. Lopp, and L. Ramroth, "FASTSim: a model to estimate vehicle efficiency, cost and performance," SAE Technical Paper, Technical Report, 2015.

[18] S. M. Casey and A. K. Lund, "Changes in speed and speed adaptation following increase in national maximum speed limit," Journal of Safety Research, vol. 23, no. 3, pp. 135-146, 1992.

[19] C. Nowakowski, S. E. Shladover, D. Cody et al., "Cooperative adaptive cruise control: testing drivers' choices of following distances," Technical Report, 2011.

[20] S. H. Fairclough, A. J. May, and C. Carter, "The effect of time headway feedback on following behaviour," Accident Analysis \& Prevention, vol. 29, no. 3, pp. 387-397, 1997.

[21] S. Shewmake and L. Jarvis, "Hybrid cars and HOV lanes," Transportation Research Part A: Policy and Practice, vol. 67, pp. 304-319, 2014.

[22] M. Chang, J. Wiegmann, A. Smith, and C. Bilotto, "A review of HOV lane performance and policy options in the United States," Federal Highway Administration, Technical Report, United States, 2008.

[23] J. A. Gomez-Ibanez, C. B. Casady, M. Fagan, J. Foote, and E. Marsh, "Toll-managed lanes: benefit-cost analyses of seven projects," Technical Report, 2018.

[24] C. Nowakowski, S. E. Shladover, and D. Cody, Cooperative Adaptive Cruise Control: Testing Drivers' Choices of Following Distances, California Partners for Advanced Transit and HIghways (PATH),Richmond, CA, USA, Technical Report, 2011.

[25] S. E. Shladover, C. Nowakowski, and X.-Y. Lu, Using Cooperative Adaptive Cruise Control (CACC) to Form High-Performance Vehicle Streams Definitions, Literature Review and Operational Concept Alternatives, Technical Report, University of California Berkeley, California Partners for Advanced Transportation Technology, 2018, https://escholarship.org/uc/item/3w6920wz.

[26] D. Swaroop, J. K. Hedrick, C. C. Chien, and P. Ioannou, "A comparision of spacing and headway control laws for automatically controlled vehicles1," Vehicle System Dynamics, vol. 23, no. 1, pp. 597-625, 1994.

[27] H. Wang, Y. Qin, W. Wang, and J. Chen, "Stability of CACCmanual heterogeneous vehicular flow with partial CACC performance degrading," Transportmetrica B: Transport Dynamics, vol. 7, no. 1, pp. 788-813, 2019.

[28] S. E. Shladover, C. Nowakowski, X.-Y. Lu, and R. Ferlis, "dCooperative Adaptive Cruise Control," Transportation 
Research Record: Journal of the Transportation Research Board, vol. 2489, no. 1, pp. 145-152, 2015.

[29] J. Larson, C. Kammer, K.-Y. Liang, and K. H. Johansson, "Coordinated route optimization for heavy-duty vehicle platoons," in Proceedings of the 2013 16th International IEEE Conference on Intelligent Transportation Systems-(ITSC), pp. 1196-1202, The Hague, Netherlands, 2013.

[30] S. Kuutti, S. Fallah, K. Katsaros, M. Dianati, F. Mccullough, and A. Mouzakitis, "A survey of the state-of-the-art localization techniques and their potentials for autonomous vehicle applications," IEEE Internet of Things Journal, vol. 5, no. 2, pp. 829-846, 2018.

[31] Z. Zhong, "Assessing the effectiveness of managed lane strategies for the rapid deployment of cooperative adaptive cruise control technology" Ph.D. thesis, Newark, NJ, USA, 2018.

[32] X. Zhang, J. Ma, B. Smith, and J. Liu, "Operational performance evaluation of the managed lane strategy for early deployment of cooperative adaptive cruise control," Technical Report, 2018.

[33] M. A. Wright, R. Horowitz, and A. A. Kurzhanskiy, "A dynamic-system-based approach to modeling driver movements across general-purpose/managed lane interfaces," in Proceedings of the ASME 2018 Dynamic Systems and Control Conference, Atlanta, GA, USA, October 2018.

[34] Z. Chen, F. He, L. Zhang, and Y. Yin, "Optimal deployment of autonomous vehicle lanes with endogenous market penetration," Transportation Research Part C: Emerging Technologies, pp. 143-156, 2016, Washington, DC, USA.

[35] Z. Zhong and J. Lee, "The effectiveness of managed lane strategies for the near-term deployment of cooperative adaptive cruise control," Transportation Research Part A: Policy and Practice, vol. 129, pp. 257-270, 2019.

[36] Z. Zhong and J. Lee, "Simulation framework for cooperative adaptive cruise control with empirical DSRC module," in Proceedings of the 44th Annual Conference of the IEEE Industrial Electronics Society, Washington, DC, USA, 2018, http://arxiv.org/abs//1810.06510.

[37] S. F. Qom, Y. Xiao, and M. Hadi, "Evaluation of Cooperative Adaptive Cruise Control (CACC) vehicles on managed lanes utilizing macroscopic and mesoscopic simulation," in Proceedings of the Transportation Research Board 95th Annual Meeting, Washington, DC, USA, 2016.

[38] A. Ghiasi, O. Hussain, Z. Qian, and X. Li, "A mixed traffic capacity analysis and lane management model for connected automated vehicles: a Markov chain method," Transportation Research Part B: Methodological, vol. 106, pp. 266-292, 2017.

[39] Z. Zhong, L. Joyoung, and L. Zhao, "Evaluations of managed lane strategies for arterial deployment of cooperative adaptive cruise control," in Proceedings of the 96th Transportation Research Board Annual Meeting, Washington, DC, USA, January 2017.

[40] A. Papadoulis, M. Quddus, and M. Imprialou, "Evaluating the safety impact of connected and autonomous vehicles on motorways," Accident Analysis \& Prevention, vol. 124, pp. 12-22, 2019.

[41] Z. Zhong, E. E. Lee, M. Nejad, and J. Lee, "Influence of CAV clustering strategies on mixed traffic flow characteristics: an analysis of vehicle trajectory data," Transportation Research Part C: Emerging Technologies, vol. 115, Article ID 102611, 2020.

[42] Y. Ali, Z. Zheng, and M. M. Haque, “eConnectivity's impact on mandatory lane-changing behaviour: evidences from a driving simulator study," Transportation Research Part C: Emerging Technologies, vol. 93, pp. 292-309, 2018.

[43] PTV Vissim, Retrieved from PTV Group, http://vision-traffic. ptvgroup.com/enus/products/ptv-vissim/use-cases/junctiongeometry, 2018.

[44] A. Kesting, M. Treiber, and D. Helbing, "Enhanced intelligent driver model to access the impact of driving strategies on traffic capacity," Philosophical Transactions of the Royal Society A: Mathematical, Physical and Engineering Sciences, vol. 368, no. 1928, pp. 4585-4605, 2010.

[45] M. Wang, S. van Maarseveen, R. Happee, O. Tool, and B. van Arem, "Benefits and risks of truck platooning on freeway operations near entrance ramp," Transportation Research Record: Journal of the Transportation Research Board, vol. 2673, no. 8, pp. 588-602, 2019.

[46] A. Kesting, M. Treiber, M. Schönhof, and D. Helbing, "Adaptive cruise control design for active congestion avoidance," Transportation Research Part C: Emerging Technologies, vol. 16, pp. 668-683, 2008.

[47] A. Talebpour, H. S. Mahmassani, and F. E. Bustamante, "Modeling driver behavior in a connected environment: integrated microscopic simulation of traffic and mobile wireless telecommunication systems," Transportation Research Record: Journal of the Transportation Research Board, vol. 2560, no. 1, pp. 75-86, 2016.

[48] A. Spiliopoulou, G. Perraki, M. Papageorgiou, and C. Roncoli, "Exploitation of acc systems towards improved traffic flow efficiency on motorways," in Proceedings of the 2017 5th IEEE International Conference on Models and Technologies for Intelligent Transportation Systems (MT-ITS), pp. 37-43, Naples, Italy, June 2017.

[49] M. Guériau, R. Billot, N.-E. E. Faouzi, J. Monteil, F. Armetta, and S. Hassas, "How to assess the benefits of connected vehicles? a simulation framework for the design of cooperative traffic management strategies," Transportation Research Part C: Emerging Technologies, vol. 67, pp. 266-279, 2016.

[50] M. Treiber, A. Hennecke, and D. Helbing, "Congested traffic states in empirical observations and microscopic simulations," Physical Review E, vol. 62, no. 2, p. 1805, 2000.

[51] S. C. Calvert, F. L. M. van Wageningen-Kessels, and S. P. Hoogendoorn, "Capacity drop through reaction times in heterogeneous traffic," Journal of Traffic and Transportation Engineering (English Edition), vol. 5, no. 2, pp. 96-104, 2018.

[52] A. Sharma, Z. Zheng, J. Kim, A. Bhaskar, and M. M. Haque, "Estimating and comparing response times in traditional and connected environments," Transportation Research Record: Journal of the Transportation Research Board, vol. 2673, no. 4, pp. 674-684, 2019.

[53] S. C. Calvert and B. van Arem, "A generic multi-level framework for microscopic traffic simulation with automated vehicles in mixed traffic," Transportation Research Part C: Emerging Technologies, vol. 110, pp. 291-311, 2020.

[54] J. W. C. van Lint and S. C. Calvert, "A generic multi-level framework for microscopic traffic simulation-Theory and an example case in modelling driver distraction," Transportation Research Part B: Methodological, vol. 117, pp. 63-86, 2018.

[55] M. Saifuzzaman, Z. Zheng, M. M. Haque, and S. Washington, "Understanding the mechanism of traffic hysteresis and traffic oscillations through the change in task difficulty level," Transportation Research Part B: Methodological, vol. 105, pp. 523-538, 2017.

[56] S. H. Hamdar, H. S. Mahmassani, and M. Treiber, "cFrom behavioral psychology to acceleration modeling: Calibration, validation, and exploration of drivers' cognitive and safety 
parameters in a risk-taking environment," Transportation Research Part B: Methodological, vol. 78, pp. 32-53, 2015.

[57] A. Sharma, Z. Zheng, A. Bhaskar, and M. M. Haque, "Modelling car-following behaviour of connected vehicles with a focus on driver compliance," Transportation Research Part B: Methodological, vol. 126, pp. 256-279, 2019.

[58] R. Fuller, "Driver control theory: from task difficulty homeostasis to risk allostasis," in Handbook of Traffic PsychologyElsevier, Amsterdam, Netherlands, 2011.

[59] M. Saifuzzaman, Z. Zheng, M. Mazharul Haque, and S. Washington, "Revisiting the Task-Capability Interface model for incorporating human factors into car-following models," Transportation Research Part B: Methodological, vol. 82, pp. 1-19, 2015.

[60] A. Bibeka, P. Songchitruksa, and Y. Zhang, "Assessing environmental impacts of ad-hoc truck platooning on multilane freeways," Journal of Intelligent Transportation Systems, vol. 0, pp. 1-12, 2019.

[61] M. Killat, F. Schmidt-Eisenlohr, H. Hartenstein et al., "Enabling efficient and accurate large-scale simulations of vanets for vehicular traffic management," in Proceedings of the Fourth ACM International Workshop on Vehicular Ad Hoc Networks, pp. 29-38, Santa Barbara, CA, USA, 2007.

[62] D. Jiang, Q. Chen, and L. Delgrossi, "Communication density: a channel load metric for vehicular communications research," in Proceedings of the 2007 IEEE International Conference on Mobile Adhoc and Sensor Systems, pp. 1-8, Pisa, Italy, October 2007.

[63] M. Killat and H. Hartenstein, "An empirical model for probability of packet reception in vehicular ad hoc networks," EURASIP Journal on Wireless Communications and Networking, vol. 2009, Article ID 721301, 2009.

[64] Leidos, Simulation of Evolutionary Introduction of Cooperative Adaptive Cruise Control Equipped Vehicles into Traffic, Saxton Transportation Operations Laboratory, Technical Report, 2016.

[65] T. Li, J. Ma, and D. K. Hale, "High-occupancy vehicle lanes on the right: an alternative design for congestion reduction at freeway merge, diverge, and weaving areas," Transportation Letters, vol. 12, no. 4, pp. 233-245, 2019.

[66] L. Xiao, M. Wang, and B. van Arem, "Traffic flow impacts of converting an HOV lane into a dedicated CACC lane on a freeway corridor," IEEE Intelligent Transportation Systems Magazine, vol. 12, pp. 60-73, 2019.

[67] Transportation Research Board, Dedicating Lanes for Priority or Exclusive Use by Connected and Automated Vehicles, The National Academies Press, Washington, DC, USA, 2018.

[68] L. A. Goodman, "Kolmogorov-Smirnov tests for psychological research," Psychological Bulletin, vol. 51, no. 2, p. 160, 1954.

[69] I. T. Young, "Proof without prejudice: use of the KolmogorovSmirnov test for the analysis of histograms from flow systems and other sources," Journal of Histochemistry \& Cytochemistry, vol. 25, no. 7, pp. 935-941, 1977.

[70] M. Gouy, K. Wiedemann, A. Stevens, G. Brunett, and N. Reed, "Driving next to automated vehicle platoons: how do short time headways influence non-platoon drivers' longitudinal control?" Transportation Research Part F: Traffic Psychology and Behaviour, vol. 27, pp. 264-273, 2014. 